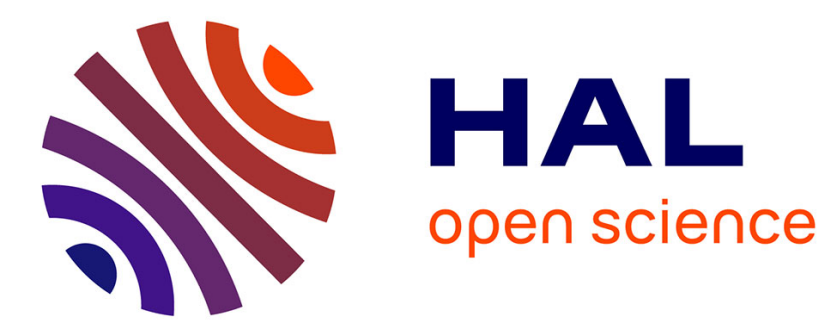

\title{
Rendezvous of Nonholonomic Robots via Output-Feedback Control under Time-varying Delays
}

Emmanuel Nuño, Antonio Loria, Elena Panteley, Esteban Restrepo

\section{To cite this version:}

Emmanuel Nuño, Antonio Loria, Elena Panteley, Esteban Restrepo. Rendezvous of Nonholonomic Robots via Output-Feedback Control under Time-varying Delays. 2021. hal-03275333

\section{HAL Id: hal-03275333 \\ https://hal.science/hal-03275333}

Preprint submitted on 1 Jul 2021

HAL is a multi-disciplinary open access archive for the deposit and dissemination of scientific research documents, whether they are published or not. The documents may come from teaching and research institutions in France or abroad, or from public or private research centers.
L'archive ouverte pluridisciplinaire HAL, est destinée au dépôt et à la diffusion de documents scientifiques de niveau recherche, publiés ou non, émanant des établissements d'enseignement et de recherche français ou étrangers, des laboratoires publics ou privés. 


\title{
Rendezvous of Nonholonomic Robots via Output-Feedback Control under Time-varying Delays
}

\author{
Emmanuel Nuño Antonio Loría Elena Panteley Esteban Restrepo
}

\begin{abstract}
In this paper we address the rendezvous problem for nonholonomic vehicles with second-order dynamics, that is, to make them converge to a formation centered at a non-prespecified point on the plane. We assume that only output feedback is available; the output corresponding to the Cartesian position of the center of mass of each vehicle and its orientations. In addition, we suppose that the robots communicate over a wireless network, so the information travels with a time-varying delay. In contrast to more common practice in output-feedback control, we do not use an observer, but the controller is dynamic. In fact, it has a clear physical interpretation of a second-order oscillator that is hinged to the actual system. Furthermore, the controller is fully distributed; for each robot it depends on the coordinates of the actual agent, the controller's states and the information received from its neighbors. We show, both analytically and via realistic GazeboROS simulations, that by exchanging the controller variables, as opposed to their own, the networked robots achieve full consensus, both in positions and orientations, in spite of the delays. To the best of our knowledge, output feedback consensus control of underactuated nonholonomic vehicles has been little studied, all the less in the presence of delays.
\end{abstract}

Index Terms-Rendezvous, consensus, autonomous vehicles, persistency of excitation, output feedback, differential-drive mobile robots.

\section{INTRODUCTION}

For first and second-order integrators the leaderless consensus problem, which consists in the state variables of all agents converging to a common value, is well-studied and solved under many different scenarios [1]. The solution to this problem is more complex if one considers the agents' dynamics [2], [3], network constraints, such as communication delays [4], unavailability of velocity measurements [5], or nonholonomic constraints that restrict the systems' motion [6].

Corresponding author: Emmanuel Nuño; Department of Computer Science at the University of Guadalajara. Guadalajara, Mexico (e-mail: emmanuel.nuno@cucei.udg.mx). A. Loría and E. Panteley are with the Laboratoire des Signaux et Systèmes, CNRS, Gif-sur-Yvette, France; (e-mail: \{antonio.loria\} \{elena.panteley\}@centralesupelec.fr). E. Panteley is also with ITMO University, St. Petersburg Russia. E. Restrepo is with DTIS, ONERA and Université Paris-Saclay, F-91123 Palaiseau, France. E-mail: esteban.restrepo@onera.fr

E. Nuño's work is supported by the Government of Mexico via the Basic Scientific Research grant CB-282807, sponsored by CONACyT. The work of A. Loría and E. Panteley is supported by the French National Research Agency (ANR) via the project "HANDY" - contract number: ANR-18-CE40-0010, and by CEFIPRA under the grant number 6001A. The work of E. Panteley is also the supported Government of the Russian Federation under grant 074-U01.
For autonomous vehicles, which, in contrast to mathematical models consisting of first and second-order integrators, do occupy a physical space, the leaderless consensus problem consists in making all robots converge to a rendezvous point. That is, the robots are required to coordinate their motions without any pre-established trajectory. Furthermore, because the robots can obviously not occupy the same physical space simultaneously, a formation pattern with an unknown center must be imposed. This is done by specifying for each robot, an offset position from the unknown center [7]. It may be required that positions and orientations converge to a common value [8], or that either only the positions [6] or only the orientations [9] achieve a common equilibrium point.

Rendezvous control is useful in cases where a group of robots must converge to postures that form a desired geometric pattern given any initial configuration in order to subsequently maneuver as a whole [10]. This is a typical two-stage formation problem. In the first, a rendezvous algorithm is required for the stabilization of the agents [11]-[13] and in the second a formation-tracking controller is employed [14]- [15].

From a systems viewpoint, rendezvous control of nonholonomic vehicles inherently is a set-point stabilization problem. In that regard, it presents the same technical difficulties as the stabilization of a single robot. In particular, that nonholonomic systems are not stabilizable via time-invariant smooth feedback [16], but either via discontinuous time-invariant control [12] or time-varying smooth feedback [17], [18]. In other words, in contrast to the case of holonomic systems, for systems with nonholonomic constraints stabilization is not a particular case of trajectory tracking, so controllers that solve one problem generally cannot solve the other [19]. For multiagent systems, necessary conditions for rendezvous are laid in [6]. Thus, neither the numerous algorithms for consensus of linear systems nor those for formation-tracking control, notably in a leader-follower configuration, apply to the rendezvous problem for nonholonomic vehicles.

In this paper we consider a rendezvous problem for secondorder (force-controlled) nonholonomic systems interconnected over an undirected static graph and with time-varying measurement delays for which velocity measurements are not available. From a systems viewpoint, this is an output-feedback control problem, with output corresponding to the vehicles' positions and orientations. We emphasize that in spite of the 
many articles on output-feedback control for the consensus of multi-agent systems, e.g., [20]-[23], very few address the problem of output-feedback control for nonholonomic vehicles; see for instance, [24] on the leader-follower consensus problem and [25] where a velocity filter has been employed to obviate the need of velocity measurements. In the latter, however, delays are not considered and, more importantly, such problem appears to be unsolvable using the algorithm proposed therein.

The main contribution of our work is the solution to the rendezvous problem via a novel smooth output feedback controller. The controller does not require velocity measurements and it is robust to interconnecting delays. Further, our controller is completely distributed because it relies only on the information available to each agent from its neighbors, without requiring any knowledge of the complete network.

The stabilization mechanism behind our proposal has a clear physical analogy with the stabilization of (under-actuated) flexible joint robots, see Section IV-B below. The controller is dynamic and achieves the control goal via a persistentlyexciting term —cf. [26].

The next section describes the dynamic model of the nonholonomic agents and, formally, describes the problem at hand. Then for clarity of exposition, in Section III, we revisit the rendezvous problem for linear second-order systems via a state-feedback controller. In Section IV we design an outputfeedback scheme for nonholonomic vehicles for the undelayed case. The main contribution of our work is presented in Section V. Section VI depicts some realistic simulations using the Gazebo-ROS environment. Finally, Section VII draws the final remarks and conclusions.

\section{MOdEL AND PROBLEM FORMULATION}

\section{A. Single-robot model}
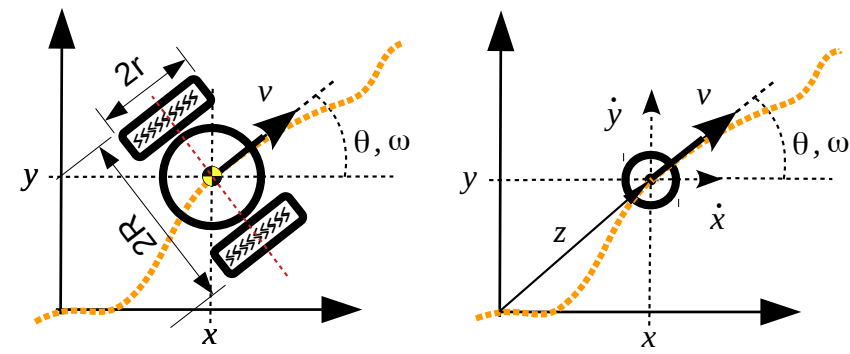

Fig. 1: Schematics of a differential-drive mobile robot.

We consider autonomous vehicles as the one schematically represented in Fig. 1. Its position on the plane may be defined as that of its center of mass, with Cartesian coordinates $(x, y) \in \mathbb{R}^{2}$ and its orientation with respect to the abscissae is denoted by the angle $\theta$. It is assumed that the vehicle may move forward with a velocity $v:=\left[\dot{x}^{2}+\dot{y}^{2}\right]^{(1 / 2)}$ and turn with an angular velocity $\omega$. The vehicle, however, cannot move in certain directions (e.g., sideways). This restriction is encoded by the non-integrable velocity constraint

$$
\sin (\theta) \dot{x}=\cos (\theta) \dot{y}
$$

From these expressions we obtain the velocity equations

$$
\begin{aligned}
\dot{x} & =\cos (\theta) v \\
\dot{y} & =\sin (\theta) v \\
\dot{\theta} & =\omega,
\end{aligned}
$$

which define a first-order model often used in the literature on control of nonholonomic systems — see e.g., [14], [27]-[29] and [15]. In such model the control inputs are the velocities $v$ and $\omega$. Being mechanical systems, however, a more complete model also includes a set of Euler-Lagrange equations for the velocity dynamics, i.e.,

$$
\begin{aligned}
\dot{v} & =F_{v}(z, \theta, v, \omega)+u_{v} \\
\dot{\omega} & =F_{\omega}(z, \theta, v, \omega)+u_{\omega},
\end{aligned}
$$

where $F_{v}$ and $F_{\omega}$ are smooth functions [30]. Articles on control of nonholonomic systems where such second-order models are used are considerably scarce in comparison - see, e.g., see [31] and [32] and they are more often found in a single-vehicle setting [33]-[35].

In this paper, we employ a complete second-order model that corresponds to that of so-called differential drive robots [36]. For the purpose of analysis, only, we assume that the center of mass is aligned with an axis joining the centers of the wheels - see the illustration on the left in Fig. 1, so $F_{\omega}$, $F_{v} \equiv 0$. The model used to test our algorithms in the realistic simulator Gazebo-ROS does not satisfy this assumption.

The control inputs take the form

$$
u_{v}:=\frac{1}{r m}\left[\tau_{1}+\tau_{2}\right], \quad u_{\omega}:=\frac{2 R}{I r}\left[\tau_{1}-\tau_{2}\right],
$$

where $m$ and $I$ are respectively the robot's mass and inertia whereas $\tau_{1}$ and $\tau_{2}$ are the torques applied, independently, at each of the wheels.

An essential feature of this model, that is at the basis of the control design, is that Equations (1)-(2) consist of two coupled second-order systems driven by independent control inputs. One system determines the linear motion and the other the angular one. To evidence this, we define $z_{i}:=\left[\begin{array}{ll}x_{i} & y_{i}\end{array}\right]^{\top} \in \mathbb{R}^{2}$, where we introduced the index $i \leq N$ to refer to one among $N$ robots - see the illustration on the right in Fig. 1, and rewrite the equations for the $i$ th robot in the form:

$$
\begin{aligned}
& \underset{\text { motion }}{\operatorname{angular}}\left\{\begin{aligned}
\dot{\theta}_{i} & =\omega_{i} \\
\dot{\omega}_{i} & =u_{\omega i},
\end{aligned}\right. \\
& \text { linear } \\
& \text { motion }\left\{\begin{aligned}
\dot{z}_{i} & =\varphi\left(\theta_{i}\right) v_{i}, \\
\dot{v}_{i} & =u_{v i},
\end{aligned}\right.
\end{aligned}
$$

where

$$
\varphi\left(\theta_{i}\right):=\left[\begin{array}{ll}
\cos \left(\theta_{i}\right) & \sin \left(\theta_{i}\right)
\end{array}\right]^{\top} .
$$

This (apparently innocuous) observation is important because the literature is rife with efficient controllers for second-order mechanical systems from which we may draw inspiration for the problem at hand here, even in the context of multi-agent systems [1]. Moreover, even though the subsystems (3) and (4) are clearly intertwined through the function $\varphi$ they may be 
dealt with as if decoupled, by replacing $\theta_{i}$ with the trajectory $\theta_{i}(t)$ since $\varphi$ is uniformly bounded [37]. Hence, relying on a cascades argument, we may apply a separation principle to design the controllers for the linear and angular motion subsystems independently. These key features are at the basis of our method to approach the rendezvous problem, which is described next.

\section{B. Problem statement: rendezvous}

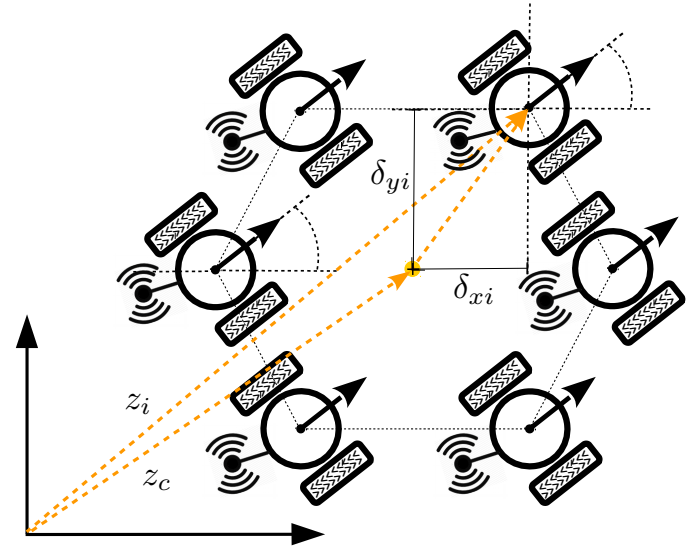

Fig. 2: Robots in formation at rendezvous

Consider a group of $N$ force-controlled nonholonomic vehicles modeled by (3)-(5) like the one depicted in Fig. 1, each of these robots is assumed to be equipped with positioning sensors that deliver reliable measures of $x_{i}, y_{i}$, and $\theta_{i}$.

The robots are required to meet in formation at a rendezvous point $z_{c}:=\left(x_{c}, y_{c}\right)$ and acquire a common orientation $\theta_{c}$. That is, for each $i \leq N$ the Cartesian positions $z_{i}$ must converge to $z_{c}+\delta_{i}$, where $\delta_{i}:=\left(\delta_{x i}, \delta_{y i}\right)$ is a vector that determines the position of the $i$ th vehicle relative to the unknown center of the formation - see Fig. 2 for an illustration. More precisely, consider the following problem statement.

Definition 1 (Rendezvous): For each robot (resp. each $i \leq$ $N)$, given a vector $\delta_{i}=\left[\begin{array}{ll}\delta_{x i} & \delta_{y i}\end{array}\right]^{\top}$, define its translated position $\bar{z}_{i}:=z_{i}-\delta_{i}$ (correspondingly, let $\bar{x}_{i}:=x_{i}-\delta_{x i}$ and $\left.\bar{y}_{i}:=y_{i}-\delta_{y i}\right)$. Then, design a distributed controller such that

$$
\begin{aligned}
& \lim _{t \rightarrow \infty} v_{i}(t)=0, \quad \lim _{t \rightarrow \infty} \bar{z}_{i}(t)=z_{c}, \\
& \lim _{t \rightarrow \infty} \omega_{i}(t)=0, \quad \lim _{t \rightarrow \infty} \theta_{i}(t)=\theta_{c} \quad \forall i \leq N .
\end{aligned}
$$

We stress that this is a leaderless consensus problem. That is, neither the coordinates $\left(x_{c}, y_{c}\right)$ nor the angle $\theta_{c}$ are determined a priori as a reference. They depend on the initial postures, the systems' nonlinear dynamics, and network features (see farther below). Stated as above, this problem, in general, may not be approached via controllers designed to make the vehicles advance in formation while following a leader (virtual or otherwise). However, the rendezvous problem has been successfully solved in a number of articles, under different conditions; some of these are cited in the Introduction. As is pointed out in this section, the originality of this paper resides in considering the scenario determined by the following hypotheses simultaneously.

Assumption 1: The velocities $v_{i}$ and $\omega_{i}$ are not measurable.

That is, only the coordinates $\left(z_{i}, \theta_{i}\right)$ are known by each robot. Then, a WiFi network is available for the robots to communicate over. Hence, the $i$ th robot communicates with a set of neighbors, which we denote by $\mathcal{N}_{i}$. It is naturally assumed that once a communication is set between two robots $i$ and $j \in \mathcal{N}_{i}$, the flow of information is bidirectional and is never lost. Therefore, the network may be modeled using an undirected time-invariant graph. Whence the following.

Assumption 2: The network may be modeled using an interconnection graph that is undirected, static, and connected.

Remark 1: In graph theory, a graph is undirected if the nodes exchange information in both direction, it is static if the interconnection is constant, and it is connected if any node is reachable from any other node [1], [38].

On the other hand, because the robots communicate through a WiFi network, the communication between the robots $i$ and $j$ is affected by non-constant time-delays. More precisely, we consider the following.

Assumption 3: The communication from the $j$ th to the $i$ th robot is subject to a variable time-delay denoted $T_{j i}(t)$ that is bounded by a known upper-bound $\bar{T}_{j i} \geq 0$ and has bounded time-derivatives, up to the second.

Assumptions 1-3 coin a realistic scenario of automatic control of multi-agent systems which has been little addressed in the literature in the context of the rendezvous problem. Assumption 3 carries certain conservatism. However, in the supposition that the delays are differentiable and bounded. Indeed, it must be stressed that, in general, time-delays over WiFi networks or the Internet may rather be of a nonsmooth nature [8], [39], [40]. Nonetheless, the formal analysis under such condition is considerably intricate and escapes the scope of this paper. For a Lyapunov-based analysis of the rendezvous problem under non-differentiable delays, albeit via state-feedback, see [8], [41].

\section{Control Architecture: State-Feedback CASE}

As previously implied, the controller that we propose relies on the system's structural properties that lead to a separation of the linear and angular motion dynamics. For clarity of exposition and to better put our contributions in perspective, we start by revisiting the consensus problem for ordinary second order systems ('double integrators') via state-feedback and without delays. The purpose is to underline the robustness of a commonly used distributed controller, by presenting an original analysis that is helpful to understand the proof of our main result.

\section{A. Consensus control of second-order systems}

The consensus problem for systems with dynamics

$$
\ddot{\vartheta}_{i}=u_{i} \quad i \leq N, u_{i} \in \mathbb{R}
$$


(that is steering $\vartheta_{i} \rightarrow \vartheta_{c}, \dot{\vartheta}_{i} \rightarrow 0$, and $\ddot{\vartheta}_{i} \rightarrow 0$ with $\vartheta_{c}$ constant and non-imposed a priori) is now well understood [1] in various settings, e.g., in the case of immeasurable velocities [42], of measurement delays [43], or with state constraints [44], [45], to mention a few.

For instance, it is well known (see [1]) that if the systems modeled by (8) communicate over a network modeled by a directed, static, and connected graph, the distributed control law, of proportional-derivative (PD) type,

$$
u_{i}=-d_{i} \dot{\vartheta}_{i}-p_{i} \sum_{j \in \mathcal{N}_{i}} a_{i j}\left(\vartheta_{i}-\vartheta_{j}\right) ; \quad d_{i}, p_{i}>0,
$$

where $a_{i j}>0$ if $j \in \mathcal{N}_{i}$ and $a_{i j}=0$ otherwise, solves the consensus problem. There are many reported ways to show this. For further development we provide here a simple and original proof based on Lyapunov's direct method. Let $\vartheta:=$ $\left[\vartheta_{1} \cdots \vartheta_{N}\right]^{\top}$ and

$$
\tilde{\vartheta}:=\vartheta-\frac{1}{N} \mathbf{1}_{N} \mathbf{1}_{N}^{\top} \vartheta, \quad \mathbf{1}_{N}:=[1 \cdots 1]^{\top} .
$$

Indeed, $\tilde{\vartheta}$ denotes a vector whose $i$ th element corresponds to the difference between $\vartheta_{i}$ and the average of all states, i.e., $\vartheta_{c}:=(1 / N) \mathbf{1}_{N}^{\top} \vartheta$. In addition, under Assumption 2, $\vartheta_{c}$ corresponds to the consensus equilibrium point. Now, to abbreviate the notation, we also define

$$
R:=I-\frac{1}{N} \mathbf{1}_{N} \mathbf{1}_{N}^{\top}
$$

Note that $R=R^{\top}$ and $\|R\| \leq 1$, where $\|R\|$ corresponds to the induced norm of $R$, and $\tilde{\vartheta}=R \vartheta$.

Next, we introduce the Laplacian matrix, $L:=\left[\ell_{i j}\right] \in$ $\mathbb{R}^{N \times N}$, where

$$
\ell_{i j}=\left\{\begin{array}{cc}
\sum_{k \in \mathcal{N}_{i}} a_{i k} & i=j \\
-a_{i j} & i \neq j .
\end{array}\right.
$$

By construction, $L \mathbf{1}_{N}=0$ and, after Assumption 2, $L$ is symmetric, it has a unique zero-eigenvalue, and all of its other eigenvalues are strictly positive. Thus, $\operatorname{rank}(L)=N-1$. Also, the last term on the right-hand side of Equation (9) satisfies

$$
\operatorname{col}\left[\sum_{j \in \mathcal{N}_{i}} a_{i j}\left(\vartheta_{i}-\vartheta_{j}\right)\right]=L \tilde{\vartheta}
$$

where $\operatorname{col}\left[(\cdot)_{i}\right]$ denotes a column vector of $N$ elements $(\cdot)_{i}$. Indeed, by the definition of the Laplacian, we have

$\operatorname{col}\left[\sum_{j \in \mathcal{N}_{i}} a_{i j}\left(\vartheta_{i}-\vartheta_{j}\right)\right]=L\left[\vartheta-\frac{1}{N} \mathbf{1}_{N} \mathbf{1}_{N}^{\top} \vartheta\right]+\frac{1}{N} L \mathbf{1}_{N} \mathbf{1}_{N}^{\top} \vartheta$.

However, $L \mathbf{1}_{N}=0$, so the right hand side of the equation above equals to $L R \vartheta$, which corresponds to $L \tilde{\vartheta}$, by definition. These identities are useful to write the closed-loop system (8)(9) in the multi-variable form

$$
\ddot{\vartheta}=-D \dot{\vartheta}-P L \tilde{\vartheta},
$$

where $P:=\operatorname{diag}\left[p_{i}\right]$ and $D:=\operatorname{diag}\left[d_{i}\right]$, and to see that the Lyapunov function

$$
V_{1}(\tilde{\vartheta}, \dot{\vartheta}):=\frac{1}{2}\left[\tilde{\vartheta}^{\top} L \tilde{\vartheta}+\dot{\vartheta}^{\top} P^{-1} \dot{\vartheta}\right]
$$

is positive definite, even if $L$ is rank deficient. Indeed, the term $\tilde{\vartheta}^{\top} L \tilde{\vartheta} \geq \lambda_{2}(L)|\tilde{\vartheta}|$, where $\lambda_{2}(L)>0$ corresponds to the second eigenvalue of $L$ (that is, the smallest positive eigenvalue), not for any $\tilde{\vartheta} \in \mathbb{R}^{N}$, but for $\tilde{\vartheta}$ as defined in (10). Now, evaluating the total derivative of $V_{1}$ along the trajectories of (13) and using $L \dot{\tilde{\vartheta}}=L \dot{\vartheta}$ (again, this holds because $L \mathbf{1}_{N}=0$ ) we see that

$$
\dot{V}_{1}(\tilde{\vartheta}, \dot{\vartheta})=\dot{\vartheta}^{\top} P^{-1} D \dot{\vartheta} .
$$

Global asymptotic stability of the consensus manifold $\{(\tilde{\vartheta}, \dot{\vartheta})=(0,0)\}$ may be ascertained from (15) by invoking Barbashin-Krasovskiı's theorem [46] (also, but wrongly, known as LaSalle's theorem). As a matter of fact, since the system is linear time-invariant, it is also globally exponentially stable and robust to external perturbations.

To see this more clearly, using $V_{1}$ it is possible to construct a simple strict Lyapunov function. This is useful to assess the robustness of system (8) in closed-loop with the consensus control law defined in (9) in terms of input-to-state stability. Let

$$
V_{2}(\tilde{\vartheta}, \dot{\vartheta}):=V_{1}(\tilde{\vartheta}, \dot{\vartheta})+\varepsilon \tilde{\vartheta}^{\top} P^{-1} \dot{\vartheta}, \quad \varepsilon \in(0,1) .
$$

In view of the properties of $V_{1}$ it is clear that $V_{2}$ also is positive definite and radially unbounded, for sufficiently small values of $\varepsilon \in(0,1)$. The total derivative of $V_{2}$ along the closed-loop trajectories yields

$$
\dot{V}_{2}(\tilde{\vartheta}, \dot{\vartheta})=\dot{V}_{1}+\varepsilon\left[\dot{\vartheta}^{\top} R P^{-1} \dot{\vartheta}-\tilde{\vartheta}^{\top} R P^{-1} D \dot{\vartheta}-\tilde{\vartheta}^{\top} L \tilde{\vartheta}\right],
$$

which, in view of (15) and the fact that $\|R\| \leq 1$, implies that

$$
\dot{V}_{2}(\tilde{\vartheta}, \dot{\vartheta}) \leq-c_{1} \frac{d_{m}}{p_{M}}|\dot{\vartheta}|^{2}-\varepsilon c_{2}|\tilde{\vartheta}|^{2}
$$

where $d_{m}$ and $p_{M}$ are the smallest and largest coefficients of $D$ and $P$ respectively, $c_{1}:=1-\varepsilon\left[\frac{1}{\lambda}+\frac{1}{d_{m}}\right]$ and $c_{2}:=\ell_{2}-\lambda \frac{d_{m}}{p_{M}}$ are positive for appropriate values of $\lambda$ and $\varepsilon \in(0,1)$ and any $\ell_{2}:=\lambda_{2}(L)>0$.

Remark 2: Consider, now, the systems $\ddot{\vartheta}_{i}=u_{i}+\alpha_{i}$ where $\alpha_{i}$ is a bounded external disturbance. Then, the previous computations lead to the inequality

$$
\dot{V}_{2}(\tilde{\vartheta}, \dot{\vartheta}) \leq-c_{1} \frac{d_{m}}{p_{M}}|\dot{\vartheta}|^{2}-\varepsilon c_{2}|\tilde{\vartheta}|^{2}+\dot{\vartheta}^{\top} \alpha
$$

with $\alpha:=\left[\alpha_{1} \cdots \alpha_{N}\right]^{\top}$. It follows that the map $\alpha \mapsto \dot{\vartheta}$ is state-strictly passive [47] and, also, the closed-loop system is input-to-state stable with respect to the input $\alpha$.

From the previous analysis, we conclude that for the angularmotion subsystem (3) the controller

$$
u_{\omega i}=-d_{\omega i} \omega_{i}-p_{i} \sum_{j \in \mathcal{N}_{i}} a_{i j}\left(\theta_{i}-\theta_{j}\right)+\alpha_{i} ; \quad d_{\omega i}, p_{\omega i}>0,
$$

ensures global asymptotic stability of the consensus manifold $\left\{\omega_{i}=0 \wedge \theta_{i}=\theta_{j}\right\}$ if $\alpha_{i} \equiv 0$ and the closed-loop system is input-to-state stable with respect to $\alpha_{i}$. We shall see farther below that such robustness is fundamental to our main results.

Remark 3: The previous computations hold with obvious changes in the notation for the angular-motion dynamics $\ddot{\theta}_{i}=$ $u_{\omega i}$, which is equivalent to (3). This will be used later. 


\section{$B$. On consensus in the linear motion}

Based on the collective knowledge recalled in Section III-A and with the purpose of designing two independent controllers for the angular and linear motion, it appears appealing to use the following control law for the subsystem (4) — cf. [48]. Let

$$
u_{v i}=-d_{v i} v_{i}-p_{v i} \varphi\left(\theta_{i}\right)^{\top} \sum_{j \in \mathcal{N}_{i}} a_{i j}\left(\bar{z}_{i}-\bar{z}_{j}\right)
$$

and let us replace the state variable $\theta_{i}$ with an arbitrary trajectory $\theta_{i}(t)$ which, for the time being we assume to be bounded and to have a bounded derivative $\omega_{i}(t)$, for all $t \geq 0$ and all $i \leq N$ (this technical assumption is relaxed later). Then, the closed-loop linear-motion dynamics (4)-(21) may be regarded as a time-varying subsystem, decoupled from the angular motion dynamics. That is,

$$
\begin{aligned}
& \dot{\bar{z}}_{i}=\varphi\left(\theta_{i}(t)\right) v_{i}, \\
& \dot{v}_{i}=-d_{v i} v_{i}-p_{v i} \varphi\left(\theta_{i}(t)\right)^{\top} \sum_{j \in \mathcal{N}_{i}} a_{i j}\left(\bar{z}_{i}-\bar{z}_{j}\right) .
\end{aligned}
$$

Next, akin to $V_{1}$ in (14), we define the Lyapunov function

$$
V_{3}(v, \bar{z}):=\frac{1}{2} \sum_{i \leq N}\left[\frac{1}{p_{v i}} v_{i}^{2}+\frac{1}{2} \sum_{j \in \mathcal{N}_{i}} a_{i j}\left(\bar{z}_{i}-\bar{z}_{j}\right)^{2}\right],
$$

where $v:=\left[v_{1} \cdots v_{N}\right]^{\top}$ and $\bar{z}:=\left[\bar{z}_{1} \cdots \bar{z}_{N}\right]^{\top}$ —cf. (12). Using the identity

$$
\frac{1}{2} \sum_{i \leq N} \sum_{j \in \mathcal{N}_{i}} a_{i j}\left(\dot{\bar{z}}_{i}-\dot{\bar{z}}_{j}\right)^{\top}\left(\bar{z}_{i}-\bar{z}_{j}\right)=\sum_{i \leq N} a_{i j} \dot{\bar{z}}_{i}^{\top}\left(\bar{z}_{i}-\bar{z}_{j}\right)
$$

- see [49] and [50, Lemma 6.1], we compute the total derivative of $V_{3}$ along the closed-loop trajectories of (22) to obtain

$$
\dot{V}_{3}(v, \bar{z})=-v^{\top} D_{v} P_{v}^{-1} v,
$$

where $P_{v}:=\operatorname{diag}\left[p_{v i}\right]$ and $D_{v}:=\operatorname{diag}\left[d_{v i}\right]$.

Now, the system in (22) being non-autonomous, BarbashinKrasovskuii's theorem does not apply, but we may use Barbălat's (Lemma A.1 in the Appendix) and Lemma A.2 to assess global asymptotic stability. To that end, we first remark that the function $V_{3}$ is positive definite and radially unbounded in $v_{i}$ and $\left|\bar{z}_{i}-\bar{z}_{j}\right|$ for all $i, j \leq N$. Then, integrating, along the trajectories, on both sides of (24) and of $\dot{V}_{3}(v(t), \bar{z}(t)) \leq 0$, we obtain that $v_{i}$ and $\left|\bar{z}_{i}-\bar{z}_{j}\right|$ are bounded, i.e., $v_{i},\left|\bar{z}_{i}-\bar{z}_{j}\right| \in \mathcal{L}_{\infty}$ and $v_{i} \in \mathcal{L}_{2}$. In addition, (24) implies that the consensus equilibrium defined by $\left\{v_{i}=0, \bar{z}_{i}=\bar{z}_{j}\right\}$ is stable. From (22), the boundedness and continuity of $\varphi\left(\theta_{i}\right)$, of $\theta_{i}(t)$, and of $\omega_{i}(t)$, we see that, also, $\dot{\bar{z}}_{i}, \dot{v}_{i}$, and, consequently, $\ddot{v}_{i} \in \mathcal{L}_{\infty}$. Since $v_{i} \in \mathcal{L}_{2} \cap \mathcal{L}_{\infty}$ and $v_{i} \in \mathcal{L}_{\infty}$ we conclude, after Lemma A.2, that $v_{i} \rightarrow 0$. Hence, since

$$
\lim _{t \rightarrow \infty} \int_{0}^{t} \dot{v}_{i}(s) d s=\lim _{t \rightarrow \infty} v_{i}(t)-v_{i}(0),
$$

we have

$$
\lim _{t \rightarrow \infty} \int_{0}^{t} \dot{v}_{i}(s) d s=-v_{i}(0)
$$

That is, the limit of $\dot{v}_{i}$ "exists and is finite" whereas the boundedness of $\ddot{v}_{i}$ implies that $\dot{v}_{i}$ is uniformly continuous.
Hence, by virtue of Barbălat's Lemma, we conclude that $\dot{v}_{i} \rightarrow 0$ as well. In turn, after (22) we see that

$$
\lim _{t \rightarrow \infty} \varphi\left(\theta_{i}(t)\right)^{\top} \sum_{j \in \mathcal{N}_{i}} a_{i j}\left(\bar{z}_{i}(t)-\bar{z}_{j}(t)\right)=0 .
$$

This expression, however, does not imply that the consensus objective is reached. Indeed, note that the set of equilibria of the system in (22) corresponds to points belonging to the set

$$
\mathcal{U}:=\left\{v_{i}=0 \wedge \varphi\left(\theta_{i}\right)^{\top} \sum_{j \in \mathcal{N}_{i}} a_{i j}\left(\bar{z}_{i}-\bar{z}_{j}\right)=0\right\},
$$

which admits points such that $\bar{z}_{i} \neq \bar{z}_{j} \in \mathbb{R}^{2}$ because $\operatorname{rank} \varphi(\theta)=1$. This means that if orientation consensus is reached and, for instance, $\theta_{i}(t) \rightarrow 0$ then $\bar{x}_{i} \rightarrow x_{c}$, but $\bar{y}_{i} \not \supset y_{c}$-see Eq. (5).

Remark 4: This shows that the consensus problem for nonholonomic systems cannot be treated as that for ordinary second-order systems like those discussed in Section III-A cf. [44], [51].

To ensure consensus it is necessary that the set of equilibria correspond to the set $\mathcal{U} \cap \mathcal{U}^{\perp}$, where

$$
\mathcal{U}^{\perp}:=\left\{v_{i}=0 \quad \wedge \quad \varphi\left(\theta_{i}\right)^{\perp \top} \sum_{j \in \mathcal{N}_{i}} a_{i j}\left(\bar{z}_{i}-\bar{z}_{j}\right)=0\right\},
$$

and

$$
\varphi\left(\theta_{i}\right)^{\perp}:=\left[\begin{array}{ll}
-\sin \left(\theta_{i}\right) & \cos \left(\theta_{i}\right)
\end{array}\right]^{\top} .
$$

That is, $\varphi\left(\theta_{i}\right)^{\perp}$ is the annihilator of $\varphi\left(\theta_{i}\right)$ hence, $\varphi\left(\theta_{i}\right)^{\perp \top} \varphi\left(\theta_{i}\right)=\varphi\left(\theta_{i}\right)^{\top} \varphi\left(\theta_{i}\right)^{\perp}=0$.

Roughly speaking, the controller must "pull" out the trajectories that may eventually get "trapped" in the set $\mathcal{U}$, whereas they do not belong to the set $\mathcal{U}^{\perp}$. To that end, we endow the angular-motion controller with a term that incorporates an external function of time (smooth and bounded) and acts as a perturbation to the angular-motion closed-loop dynamics. This perturbation is designed to persist as long as

$$
\varphi\left(\theta_{i}\right)^{\perp \top} \sum_{j \in \mathcal{N}_{i}} a_{i j}\left(\bar{z}_{i}-\bar{z}_{j}\right) \neq 0 .
$$

More precisely, let $\psi_{i}, \dot{\psi}_{i}$, and $\ddot{\psi}_{i}$ be bounded (belong to $\mathcal{L}_{\infty}$ ) let $\dot{\psi}_{i}$ be persistently exciting [52], that is, let there exist $T$ and $\mu>0$ such that

$$
\int_{t}^{t+T} \psi_{i}(s)^{2} d s \geq \mu \quad \forall t \geq 0 .
$$

Then, for the control law in (20), we define

$$
\alpha_{i}\left(t, \theta_{i}, \bar{z}_{i}\right):=k_{\alpha i} \psi_{i}(t) \varphi_{i}\left(\theta_{i}\right)^{\perp \top}\left(\bar{z}_{i}-\bar{z}_{j}\right), \quad k_{\alpha i}>0 .
$$

The following remarks are in order.

- While $\alpha_{i}$ injects excitation into the system, which ensures that the position consensus errors converge, it acts as a bounded (hence harmless) perturbation on the angularmotion dynamics — cf. Section III-A.

- The state-feedback controller defined by (20), (21), and (27) ensures full consensus, in position and orientation, for the closed-loop system, even in the presence of delays [7]. 
- Controllers for nonholonomic systems that make explicit use of persistency of excitation were first used for tracking in [53] and for set-point stabilization in [26], but the underlying ideas are already present in [54], [55]. Nowadays, persistency of excitation is recognized as a fundamental, if not necessary, condition [19], for set-point stabilization of nonholonomic systems via smooth feedback and they are also frequently used in trajectory-tracking scenarii - see e.g., [56], [57].

\section{Control architecture: output-FEedback CASE}

We have argued that the consideration of the nonholonomic system's dynamics as being constituted by an angular and a linear-motion dynamics leads to the control design for each of these subsystems separately. This was demonstrated in the case of state-feedback control. In this section we build upon the previous analyses and designs and show how a simple, linear, dynamic output-feedback, controller may be designed to achieve consensus both in position and orientation.

\section{A. Output-feedback consensus: the rationale behind}

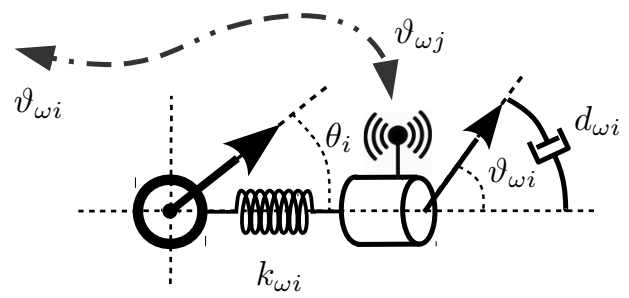

Fig. 3: Schematic representation of coupled mass-spring-damper systems: angular motion. It is the controller state variable, $\vartheta_{\omega i}$ that is transmitted to neighboring robots and, correspondingly, $\vartheta_{\omega j}$ is received from $\mathcal{N}_{i}$ neighbors.

Let us consider, first, the angular-motion dynamics, (3). Note that, expressed as $\ddot{\theta}_{i}=u_{\omega i}$, this system corresponds to an elementary Newtonian force-balance equation with unitary inertia. The problem at hand still is to synchronize the angular positions $\theta_{i}$ for $N$ such systems, but since $\omega_{i}$ is not available from measurement, we cannot use the control law in (20) with $\alpha_{i} \equiv 0-$. Yet, it appears reasonable to conjecture that the objective $\theta_{i} \rightarrow \theta_{j}$ for all $i, j \leq N$ may be achieved by coupling the subsystems $\ddot{\theta}_{i}=u_{\omega i}$, via torsional springs, to virtual second-order oscillators for which the states are available and are synchronized by design - see Fig. 3 for an illustration.

More precisely, consider the dynamic system

$$
\ddot{\vartheta}_{\omega i}+d_{\omega i} \dot{\vartheta}_{\omega i}+p_{\omega i} \sum_{j \in \mathcal{N}_{i}} a_{i j}\left(\vartheta_{\omega i}-\vartheta_{\omega j}\right)=\nu_{\omega i}
$$

where $\nu_{\omega i}$ is an external input to be defined, the state $\vartheta_{\omega i} \in \mathbb{R}$, and $d_{\omega i}, p_{\omega i}>0$.

As we showed in Section III-A, for (28) consensus is achieved, that is, there exists a real constant $\vartheta_{\omega c}$, such that $\vartheta_{\omega i} \rightarrow \vartheta_{\omega c}, \dot{\vartheta}_{\omega i} \rightarrow 0$, for all $i \leq N$, provided that $d_{\omega i}$, $p_{\omega i}>0$, and $\nu_{\omega i}=0$. On the other hand, the system in (28) defines a passive map $\nu_{\omega i} \mapsto \dot{\vartheta}_{\omega i}$. Furthermore, the system (3b) also defines a passive map, $u_{\omega i} \mapsto \omega_{i}$. Therefore, it results natural to "hitch" the systems (28) and (3) by setting $\nu_{\omega i}:=-u_{\omega i}$ and

$$
u_{\omega i}:=-k_{\omega i}\left(\theta_{i}-\vartheta_{\omega i}\right), \quad k_{\omega i}>0 .
$$

That is, the coupling $-k_{\omega i}\left(\theta_{i}-\vartheta_{\omega i}\right)$ may be interpreted as the force exerted by a torsional spring that hinges the (angular) positions of the two subsystems - again, see Fig. 3.

Remark 5: Some readers will recognize here an analogy with flexible-joint manipulators [58], [59]. This is discussed in further detail in the next section.

Thus, we have the following.

Proposition 1 (Output feedback orientation consensus):

Consider the system (3) in closed-loop with the dynamic controller defined by (28), (29), and $\nu_{\omega i}:=k_{\omega i}\left(\theta_{i}-\vartheta_{\omega i}\right)$. Let Assumption 2 hold. Then, there exist constants $\theta_{c}$ and $\vartheta_{c} \in \mathbb{R}$ such that, for all $i$ and $j \leq N$,

$$
\begin{array}{ll}
\lim _{t \rightarrow \infty} \theta_{i}(t)=\lim _{t \rightarrow \infty} \theta_{j}(t)=\theta_{c}, & \lim _{t \rightarrow \infty} \omega_{i}(t)=0, \\
\lim _{t \rightarrow \infty} \vartheta_{\omega i}(t)=\lim _{t \rightarrow \infty} \vartheta_{\omega j}(t)=\vartheta_{c}, & \lim _{t \rightarrow \infty} \dot{\vartheta}_{j}(t)=0 .
\end{array}
$$

Proof: Consider the function

$$
W_{3}\left(\dot{\vartheta}_{\omega}, \vartheta_{\omega}, \theta, \omega\right):=W_{1}\left(\vartheta_{\omega}, \dot{\vartheta}_{\omega}\right)+W_{2}\left(\theta, \omega, \vartheta_{\omega}\right),
$$

where $\vartheta_{\omega}:=\left[\vartheta_{\omega 1} \cdots \vartheta_{\omega N}\right]^{\top}$,

$$
\begin{aligned}
W_{1}\left(\vartheta_{\omega}, \dot{\vartheta}_{\omega}\right) & :=\frac{1}{2} \sum_{i \leq N}\left[\frac{\dot{\vartheta}_{i}^{2}}{p_{\omega i}}+\frac{1}{2} \sum_{j \in \mathcal{N}_{i}} a_{i j}\left(\vartheta_{\omega i}-\vartheta_{\omega j}\right)^{2}\right], \\
W_{2}(\theta, \omega, \vartheta) & :=\frac{1}{2} \sum_{i \leq N}\left[\frac{\omega_{i}^{2}}{p_{\omega i}}+k_{\omega i}\left(\theta_{i}-\vartheta_{\omega i}\right)^{2}\right] .
\end{aligned}
$$

The function $W_{2}$ corresponds to the total energy of the massspring (closed-loop) system $\ddot{\theta}_{i}=-k_{\omega i}\left(\theta_{i}-\vartheta_{\omega i}\right)$; the first term is the kinetic energy and the second the potential energy "stored" in the torsional spring of stiffness $k_{\omega i}$. The function $W_{1}$ is analogous to $V_{3}$ in (23). The function $W_{3}$ is positive definite in the consensus errors, in $\omega, \vartheta_{\omega}$, and $\dot{\vartheta}_{\omega}$. Moreover, its total derivative along the trajectories of the closed-loop system yields

$$
\dot{W}_{3}\left(\dot{\vartheta}_{\omega}, \vartheta_{\omega}, \theta, \omega\right)=-\frac{1}{2} \sum_{i \leq N} \frac{d_{\omega i}}{p_{\omega i}} \dot{\vartheta}_{\omega i}^{2} .
$$

Then, the system being autonomous, we may invoke Barbashin-Krasovskii's theorem. First, we see that $\dot{W}_{3}=0$ if and only if $\dot{\vartheta}_{\omega i}=0$. The latter implies that $\ddot{\vartheta}_{\omega i}=0$ and $\vartheta_{\omega i}=$ const for all $i \leq N$. From (28) and $\nu_{\omega i}:=k_{\omega i}\left(\theta_{i}-\vartheta_{\omega i}\right)$ we conclude that $\theta_{i}=$ const, i.e., $\omega_{i}=\dot{\omega}_{i}=0$. In turn, from $\dot{\omega}_{i}=-k_{\omega i}\left(\theta_{i}-\vartheta_{\omega i}\right)=-\nu_{\omega i}=0$ and (28) it follows that

$$
\sum_{j \in \mathcal{N}_{i}} a_{i j}\left(\vartheta_{\omega i}-\vartheta_{\omega j}\right)=0 \quad \text { and } \quad \theta_{i}=\vartheta_{\omega i} \forall i, j \leq N .
$$

Finally, in view of Assumption 2. The only solution to the equations above is $\theta_{i}=\vartheta_{\omega i}=\vartheta_{c}$ for all $i, j \leq N$. 


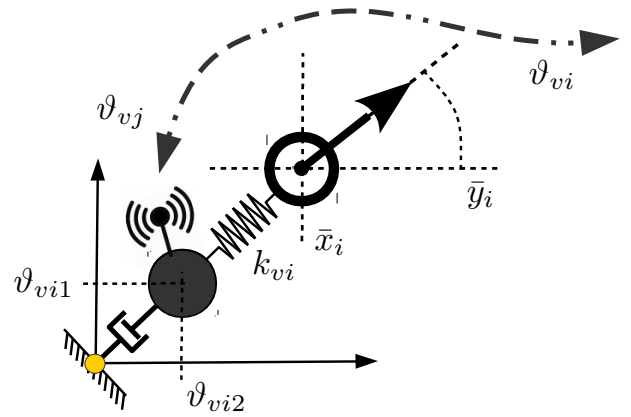

Fig. 4: Schematic representation of coupled mass-spring-damper systems: linear motion. The controller state variable $\vartheta_{v i}$ is sent to neighboring robots and, correspondingly, $\vartheta_{v j}$ is received from $\mathcal{N}_{i}$ neighbors.

\section{B. Output-feedback position consensus}

Akin to the controller for the angular motion subsystem, to steer the Cartesian positions $\bar{z}_{i}$ to a consensual point on the plane $z_{c}$, we use a second-order dynamic controller system that is reminiscent of the equation (8) in closed loop with the control (9), and an added virtual-spring coupling term, $-k_{v i}\left(\vartheta_{v i}-\bar{z}_{i}\right)$. That is, let

$$
\ddot{\vartheta}_{v i}+d_{v i} \dot{\vartheta}_{v i}+p_{v i} \sum_{j \in \mathcal{N}_{i}} a_{i j}\left(\vartheta_{v i}-\vartheta_{v j}\right)=-k_{v i}\left(\vartheta_{v i}-\bar{z}_{i}\right) \text {, }
$$

where $\vartheta_{v i} \in \mathbb{R}^{2}$ and $\dot{\vartheta}_{v i}$ are controller's state variables, and all control gains $d_{v i}, p_{v i}$ and $k_{v i}$ are positive.

Then, the dynamical system (33) is coupled to the double (nonholonomic) integrator (4). In contrast to the case of the angular motion, however, for the linear motion the control input $u_{v i}$ must incorporate the change of coordinates defined by $\varphi$. Therefore, we define

$$
u_{v i}:=-\varphi\left(\theta_{i}\right)^{\top} k_{v i}\left(\bar{z}_{i}-\vartheta_{v i}\right), \quad k_{v i}>0
$$

\section{—cf. Eq. (29).}

The following remarks are in order

- The control design is based on the ability to separate the linear and angular motion dynamics. As it is discussed in Section III-B, however, this is only an artifice for the purpose of analysis (and to rationalize the control design). Nonetheless, the systems (3) and (4) in closed loop with the controllers defined by (28) with $\nu_{\omega i}:=k_{\omega i}\left(\theta_{i}-\vartheta_{\omega i}\right)$ and (29), as well as (33), (34) cannot be expected to reach consensus unless the control input (29) is redesigned, enhanced with a $\delta$-persistently exciting term $\alpha_{i}$. This problem is addressed in the next section.

- There is an interesting analogy between the control architecture proposed above and (consensus) control of robot manipulators with flexible joints. To better see this, consider the Euler-Lagrange equations for such systems, in closedloop with a proportional-derivative consensus controller like the one defined in (9). We have — see [58], [59]—

$$
\begin{aligned}
& M_{i}\left(q_{1 i}\right) \ddot{q}_{1 i}+C_{i}\left(q_{1 i}, \dot{q}_{1 i}\right) \dot{q}_{1 i}+g_{i}\left(q_{1 i}\right)=K\left(q_{2 i}-q_{1 i}\right) \\
& \ddot{q}_{2 i}+K\left(q_{2 i}-q_{1 i}\right)=d_{i} \dot{q}_{2 i}+p_{i} \sum_{j \in \mathcal{N}_{i}} a_{i j}\left(q_{2 i}-q_{2 j}\right)
\end{aligned}
$$

and consensus is reached provided that $d_{i}, p_{i}>0$; this follows from the main results in [60].

In (35), the generalized coordinates $q_{2 i}$ correspond to the actuators' angular positions while $q_{1 i}$ correspond to the links' positions. Hence, in the case of the angular motion dynamics, the closed-loop equation $\ddot{\theta}_{i}=-k_{\omega i}\left(\theta_{i}-\vartheta_{\omega i}\right)$ may be assimilated to Eq. (35a) with unitary inertia $M_{i}=I$ and null Coriolis and gravitational forces, i.e., $C_{i}=g_{i} \equiv 0$. On the other hand, the dynamic controller (28) with $\nu_{\omega i}:=$ $k_{\omega i}\left(\theta_{i}-\vartheta_{\omega i}\right)$ corresponds, up to obvious changes in the notation, to the actuator dynamics in closed loop, that is, Eq. (35b).

These observations are significant, on one hand, because they illustrate the underlying stabilization mechanism of our approach and, on the other, as they provide guidelines beyond Section III-A to construct suitable energy-like Lyapunov functions for the analysis of the overall system. For further details, see Section V-B.

\section{OUTPUT FEEDBACK CONTROL UNDER DELAYS}

In this section we present our main result, a dynamic output feedback controller under delayed measurements. It is designed following the certainty-equivalence principle and the previous developments. For clarity of exposition, only a sketch of proof of the main statement is provided here, but complementary details are presented in the Appendix.

\section{A. Main result}

In the presence of measurement delays $T_{j i}(t)$ (see Assumption 3), which are different for each pair of robots, the consensus errors take the following forms. For the position errors, we have

$$
e_{v i}:=\sum_{j \in \mathcal{N}_{i}} a_{i j}\left(\vartheta_{v i}-\vartheta_{v j}\left(t-T_{j i}(t)\right)\right),
$$

whereas for the orientations,

$$
e_{\omega i}:=\sum_{j \in \mathcal{N}_{i}} a_{i j}\left(\vartheta_{\omega i}-\vartheta_{\omega j}\left(t-T_{j i}(t)\right)\right) .
$$

Remark 6: Note that in both cases, as in previous sections, the errors are defined in the controllers' coordinates and not on robots' measured variables.

Based on (34) and (33), the certainty-equivalence controller for the linear motion dynamics, (4), is given by

$$
\begin{aligned}
& u_{v i}=-k_{v i} \varphi\left(\theta_{i}\right)^{\top}\left(\bar{z}_{i}-\vartheta_{v i}\right), \\
& \ddot{\vartheta}_{v i}=-d_{v i} \dot{\vartheta}_{v i}-k_{v i}\left(\vartheta_{v i}-\bar{z}_{i}\right)-p_{v i} e_{v i},
\end{aligned}
$$

whereas, for the angular motion dynamics, we introduce

$$
\begin{aligned}
& u_{\omega i}=-k_{\omega i}\left(\theta_{i}-\vartheta_{\omega i}\right)+\alpha_{i}\left(t, \theta_{i}, e_{v i}\right), \\
& \ddot{\vartheta}_{\omega i}=-d_{\omega i} \dot{\vartheta}_{\omega i}-k_{\omega i}\left(\vartheta_{\omega i}-\theta_{\omega i}\right)-p_{\omega i} e_{\omega i},
\end{aligned}
$$

All constant parameters are defined as above. In addition, in order to be used with an output-feedback controller, the function $\alpha_{i}$ is redefined —cf. Eq. (27) — as

$$
\alpha_{i}\left(t, \theta_{i}, e_{v i}\right):=k_{\alpha i} \psi_{i}(t) \varphi\left(\theta_{i}\right)^{\perp \top}\left(\vartheta_{v i}-\bar{z}_{i}\right),
$$


where $k_{\alpha i}>0, \psi_{i}$ is twice differentiable, bounded, with bounded derivatives and $\dot{\psi}_{i}$ is persistently exciting —cf. Section III-B. That is, $\alpha_{i}$ in (39a) fulfills the same role as explained above. Then, we have the following.

Proposition 2: Consider the system (3)-(5), under Assumptions $1-3$, in closed-loop with (38)-(40). Then, the leaderless consensus control goal is achieved, that is, (6) and (7) hold provided that

$$
\begin{aligned}
d_{v i} & >\frac{1}{2} p_{v i} \sum_{j \in \mathcal{N}_{i}} a_{i j}\left[\beta_{i}+\frac{\bar{T}_{j i}^{2}}{\beta_{j}}\right] \\
d_{\omega i} & >\frac{1}{2} p_{\omega i} \sum_{j \in \mathcal{N}_{i}} a_{i j}\left[\varepsilon_{i}+\frac{\bar{T}_{j i}^{2}}{\varepsilon_{j}}\right]
\end{aligned}
$$

for some $\beta_{i}, \varepsilon_{i}>0$, for all $i \leq N$.

\section{B. Sketch of proof of Proposition 2}

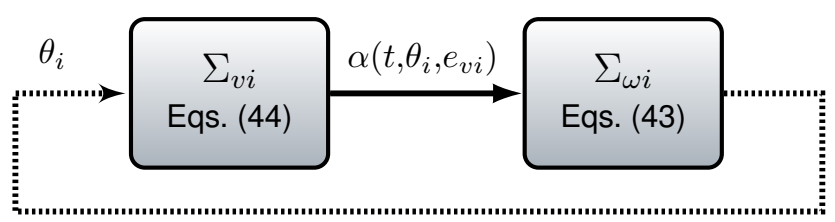

Fig. 5: Schematic representation of the closed-loop system, consisting in the error equations for the angular and the linear-motion dynamics. Even though the systems are feedback interconnected, for the purpose of analysis, they may be regarded as in cascade [37], whence the feedback represented by a dashed arrow.

We start by laying out the closed-loop equations, according to the logic of separating the linear and the angular-motion dynamics. After a direct computation, we obtain

$$
\begin{aligned}
& \Sigma_{\omega i}:\left\{\begin{array}{l}
\dot{\theta}_{i}=\omega_{i} \\
\dot{\omega}_{i}=-k_{\omega i}\left(\theta_{i}-\vartheta_{\omega i}\right)+\alpha_{i}\left(t, \theta_{i}, e_{v i}\right) \\
\ddot{\vartheta}_{\omega i}=-d_{\omega i} \dot{\vartheta}_{\omega i}-k_{\omega i}\left(\vartheta_{\omega i}-\theta_{i}\right)-p_{\omega i} e_{\omega i}
\end{array}\right. \\
& \Sigma_{v i}:\left\{\begin{array}{l}
\dot{\bar{z}}_{i}=\varphi\left(\theta_{i}\right) v_{i} \\
\dot{v}_{i}=-k_{v i} \varphi\left(\theta_{i}\right)^{\top}\left(\bar{z}_{i}-\vartheta_{v i}\right) \\
\ddot{\vartheta}_{v i}=-d_{v i} \dot{\vartheta}_{v i}-k_{v i}\left(\vartheta_{v i}-\bar{z}_{i}\right)-p_{v i} e_{v i}
\end{array}\right.
\end{aligned}
$$

As illustrated in Fig. 5, for each robot, the closed-loop equations consist of two dynamical feedback-interconnected systems. However, as explained in Section III-B, by replacing the state variables $\theta_{i}$ with fixed, but arbitrary, trajectories $\theta_{i}(t)$ in (44a) and (44b), these systems may be considered as if interconnected in cascade. Then, the analysis of $\Sigma_{\omega i}$ and $\Sigma_{v i}$ may be carried out using arguments for such systems [37]. In a nutshell, one needs to establish that: 1) all trajectories are bounded; 2) for $\Sigma_{\omega i}$ with $\alpha_{i} \equiv 0$ the consensus errors converge to zero - cf. Proposition 1;3) For $\Sigma_{v i}$ the consensus errors converge, under the persistently exciting effect of $\alpha_{i}$.

A more formal proof is provided below. For clarity of exposition, it is articulated into a sequence of claims and pertinent observations that may be drawn from them. All these are organized in two subsections pertaining to the boundedness and the convergence of the error trajectories. Details are relegated to the Appendix.

\section{1) All the trajectories are bounded:}

Claim 1: For the system $\Sigma_{v i}$ in (44), the following holds true: $\dot{\vartheta}_{v i} \in \mathcal{L}_{2}$ whereas $v_{i}, \dot{\vartheta}_{v i},\left|\vartheta_{v i}-\bar{z}_{i}\right|$, and $\left|\vartheta_{v i}-\vartheta_{v j}\right|$, all belong to $\mathcal{L}_{\infty}$ (are bounded). Furthermore, also $e_{v i} \in \mathcal{L}_{\infty}$ and, consequently, $\ddot{\vartheta}_{v i} \in \mathcal{L}_{\infty}$ too. Finally, $\dot{\vartheta}_{v i} \rightarrow 0$.

The proof of Claim 1 is provided in the Appendix. It relies on standard signal chasing analysis [61] and on the energy-based Lyapunov-Krasovskĭi functional defined below

$$
\begin{aligned}
\mathcal{V} & :=\sum_{i \leq N}\left[\frac{1}{p_{v i}} \mathcal{E}_{i}+\frac{1}{4} \sum_{j \in \mathcal{N}_{i}} a_{i j}\left|\vartheta_{v i}-\vartheta_{v j}\right|^{2}+\Upsilon_{i}^{v}\right] \\
\mathcal{E}_{i} & :=\frac{1}{2}\left[v_{i}^{2}+\left|\dot{\vartheta}_{v i}\right|^{2}+k_{v i}\left|\vartheta_{v i}-\bar{z}_{i}\right|^{2}\right] \\
\Upsilon_{i}^{v} & :=\frac{1}{2 \beta_{i}} \sum_{j \in \mathcal{N}_{i}} a_{i j} \bar{T}_{j i} \int_{-\bar{T}_{j i}}^{0} \int_{t+\eta}^{t}\left|\dot{\vartheta}_{v j}(\sigma)\right|^{2} d \sigma d \eta,
\end{aligned}
$$

where $\beta_{i}>0$ is introduced in the statement of Proposition 2. The function $\mathcal{E}_{i}$ is reminiscent of an energy function; the first two quadratic-in-velocities terms on the right-hand side may be assimilated to kinetic energy terms while the third corresponds to a potential-energy term associated to the springs with stiffness $k_{v i}$-see Fig. 4 . The second term in the definition of $\mathcal{V}$ is quadratic in the consensus errors and it is equivalent to $\tilde{\vartheta}_{v i}^{\top} L \tilde{\vartheta}_{v i}$ with $\tilde{\vartheta}_{v i}$ defined as in (9) —cf. $V_{1}$ in (14). Finally, because the closed-loop system is in the form of a set of functional differential equations, we use a so-called Lyapunov-Krasovskĭi functional [62], that is the term $\Upsilon_{i}^{v}$.

Now, the total derivative of $\mathcal{V}$ along the trajectories of (44) yields

$$
\dot{\mathcal{V}} \leq-\sum_{i \leq N}\left[\frac{d_{v i}}{p_{v i}}-\sum_{j \in \mathcal{N}_{i}} a_{i j}\left[\frac{\beta_{i}}{2}+\frac{\bar{T}_{j i}^{2}}{2 \beta_{j}}\right]\right]\left|\dot{\vartheta}_{v i}\right|^{2}
$$

— see the Appendix for details. Note that the factor of $\left|\dot{\vartheta}_{v i}\right|^{2}$ for any $i \leq N$ is positive under condition (41). The statement of the claim follows from integrating along trajectories on both sides of (46) and invoking Lemmata A.1 and A.2 in the Appendix.

Next, we analyze the behavior of the trajectories of $\Sigma_{\omega}$ and, in first instance, we assume that $\alpha_{i} \equiv 0$; this convenient hypothesis is relaxed farther below. We start with the following.

Claim 2: Under the condition that $\alpha_{i} \equiv 0$ the trajectories of $\Sigma_{\omega i}$ satisfy the following: $\dot{\vartheta}_{\omega i} \in \mathcal{L}_{2} \cap \mathcal{L}_{\infty}$ whereas $\omega_{i}$, $\left[\vartheta_{\omega i}-\theta_{i}\right],\left[\vartheta_{\omega i}-\vartheta_{\omega j}\right]$, and $e_{\omega i} \in \mathcal{L}_{\infty}$. Finally, $\dot{\vartheta}_{\omega i} \rightarrow 0$ asymptotically.

Similarly to the proof of Claim 1, the proof of Claim 2 relies on the Lyapunov-Krasovskii functional

$$
\begin{aligned}
\mathcal{W} & :=\sum_{i \leq N}\left[\frac{1}{p_{\omega i}} \mathcal{H}_{i}+\frac{1}{4} \sum_{j \in \mathcal{N}_{i}} a_{i j}\left(\vartheta_{\omega i}-\vartheta_{\omega j}\right)^{2}+\Upsilon_{i}^{\omega}\right] \\
\mathcal{H}_{i} & :=\frac{1}{2}\left[\omega_{i}^{2}+\dot{\vartheta}_{\omega i}^{2}+k_{\omega i}\left(\vartheta_{\omega i}-\theta_{i}\right)^{2}\right],
\end{aligned}
$$

where $\varepsilon_{i}>0$-cf. Eq. (45c) and $\Upsilon_{i}^{\omega}$ is defined as in (45c), replacing $\beta_{i}$ with $\varepsilon_{i}$ and $\vartheta_{v j}$ with $\vartheta_{\omega j}$. Its derivative along the trajectories of $\Sigma_{\omega i}$ with $\alpha_{i} \equiv 0$ satisfies (see the Appendix for 
details)

$$
\dot{\mathcal{W}} \leq-\sum_{i \leq N}\left[\frac{d_{\omega i}}{p_{\omega i}}-\sum_{j \in \mathcal{N}_{i}} a_{i j}\left[\frac{\varepsilon_{i}}{2}+\frac{\bar{T}_{j i}^{2}}{2 \varepsilon_{j}}\right]\right] \dot{\vartheta}_{\omega i}^{2},
$$

in which the factor of $\dot{\vartheta}_{\omega i}^{2}$ is positive in view of the condition (42). The first part of the Claim follows by integrating on both sides of the inequality in (48). Then, a simple inspection of (43) reveals that $\dot{\omega}_{i}$ and $\ddot{\vartheta}_{\omega i}$ are also bounded (belong to $\mathcal{L}_{\infty}$ ), so after Lemma A.2 it follows that $\lim _{t \rightarrow \infty} \dot{\vartheta}_{\omega i}(t)=0$.

In addition, the following assertion is established in the Appendix.

Claim 3: In the case that $\left\{\alpha_{i} \equiv 0\right\}$ it holds that $\omega_{i} \rightarrow 0$,

$$
\lim _{t \rightarrow \infty} \theta_{i}(t)=\theta_{c}
$$

and

$$
\lim _{t \rightarrow \infty} \theta_{i}(t)-\vartheta_{\omega i}(t)=0 .
$$

Now, the previous statements regarding $\Sigma_{\omega}$ hold under the restriction that $\alpha_{i} \equiv 0$. If this is not the case, we remark that since, $\psi_{i}, \vartheta_{v i}-\bar{z}_{i}$ and $\varphi^{\perp}$ are bounded along all trajectories, so is $\alpha_{i}$-see Eq. (40). This and the fact that $\Sigma_{\omega}$ is a marginally stable linear time-varying system with uniformly bounded time-delays, imply that $\dot{\omega}_{i}, \omega_{i}, \dot{\vartheta}_{\omega i}, \ddot{\vartheta}_{\omega i} \in \mathcal{L}_{\infty}$ and so $\theta_{i}-\vartheta_{\omega i}$ and $e_{\omega i}$ are also bounded —cf. [63, Proposition 3].

\section{2) The consensus errors vanish:}

So far, we have assessed the boundedness of $\left|\theta_{i}-\vartheta_{\omega i}\right|, \omega_{i}$, $\dot{\omega}_{i}, \dot{\vartheta}_{\omega i}, \ddot{\vartheta}_{\omega i}$, and $e_{\omega i}$, as well as of $v_{i}, \dot{\vartheta}_{v i},\left[\vartheta_{v i}-\bar{z}_{i}\right]$, and $\left|\vartheta_{v i}-\vartheta_{v j}\right|$. In addition, we established that $\dot{\vartheta}_{v i} \in \mathcal{L}_{2}$ and $\dot{\vartheta}_{v i} \rightarrow 0$. Next, we establish the limits in (6) and (7).

The limits in (7) follow from the marginal stability of $\Sigma_{\omega i}$ with $\alpha_{i} \equiv 0$ and, once more, invoking —cf. [63, Proposition 3], provided that $\alpha_{i} \rightarrow 0$. The latter, in turn, holds in view of the following assertion whose proof is presented in the Appendix.

Claim 4: The term $\left(\bar{z}_{i}-\vartheta_{v i}\right)$ converges to zero asymptotically.

Now, in regards to the first limit in (6) we argue as follows. Since $\varphi$ is uniformly bounded, from (44a) and (44b) we have that $\dot{\bar{z}}_{i} \in \mathcal{L}_{\infty}$ and $\dot{v}_{i} \in \mathcal{L}_{\infty}$. Moreover, from Assumption 3 and the fact that $\ddot{\vartheta}_{v i}, \dot{\vartheta}_{v i}, \dot{\bar{z}}_{i} \in \mathcal{L}_{\infty}$ it holds that

$$
\vartheta_{v i}^{(3)}=-d_{v i} \ddot{\vartheta}_{v i}-k_{v i}\left(\dot{\vartheta}_{v i}-\dot{\bar{z}}_{i}\right)-p_{v i} \dot{e}_{v i}
$$

is bounded too. This last and

$$
\lim _{t \rightarrow \infty} \int_{0}^{t} \ddot{\vartheta}_{v i}(\sigma) d \sigma=\lim _{t \rightarrow \infty} \dot{\vartheta}_{v i}(t)-\dot{\vartheta}_{v i}(0)=-\dot{\vartheta}_{v i}(0)
$$

imply, by Barbălat's Lemma, that $\lim _{t \rightarrow \infty} \ddot{\vartheta}_{v i}(t)=0$. Furthermore, we note that also $\vartheta_{v i}^{(4)} \in \mathcal{L}_{\infty}$, where

$$
\vartheta_{v i}^{(4)}=-d_{v i} \vartheta_{v i}^{(3)}-k_{v i}\left(\ddot{\vartheta}_{v i}-\ddot{\bar{z}}_{i}\right)-p_{v i} \ddot{e}_{v i} .
$$

To see this, note that since $\ddot{\bar{z}}_{i}=\varphi\left(\theta_{i}\right) \dot{v}_{i}+\omega_{i} \varphi^{\perp}\left(\theta_{i}\right) v_{i}$ and we have previously established that $\omega_{i} \in \mathcal{L}_{\infty}$ then $\vartheta_{v i}^{(4)} \in \mathcal{L}_{\infty}$ as required. Thus, $\vartheta_{v i}^{(3)}$ is uniformly continuous and tends to zero (after Barbălat's Lemma A.1). From (51) it also follows that $\dot{\bar{z}}_{i} \rightarrow 0$. Hence $\lim _{t \rightarrow \infty} v_{i}(t)=0$, which corresponds to the first limit in (6).

Remark 7: For further development we stress that $\dot{v}_{i}, \ddot{v}_{i} \rightarrow$ 0 . To see this, note that

$$
\lim _{t \rightarrow \infty} \int_{0}^{t} \dot{v}_{i}(\sigma) d \sigma=\lim _{t \rightarrow \infty} v_{i}(t)-v_{i}(0)=-v_{i}(0)
$$

and since all signals in the right-hand side of

$$
\ddot{v}_{i}=-k_{v i} \varphi\left(\theta_{i}\right)^{\top}\left(\dot{\bar{z}}_{i}-\dot{\vartheta}_{v i}\right)-k_{v i} \omega_{i} \varphi\left(\theta_{i}\right)^{\perp \top}\left(\bar{z}_{i}-\vartheta_{v i}\right)
$$

are bounded, then $\ddot{v}_{i} \in \mathcal{L}_{\infty}$ so $\dot{v}_{i}$ is uniformly continuous and Barbălat's Lemma implies that $\lim _{t \rightarrow \infty} \dot{v}_{i}(t)=0$. Following a similar argument, it also follows that $\lim _{t \rightarrow \infty} \ddot{v}_{i}(t)=0$.

The second limit in (6) is implied by $\dot{\vartheta}_{v i}, \ddot{\vartheta}_{v i}$, and $\left[\bar{z}_{i}-\vartheta_{v i}\right]$ converging to zero (for the latter see Claim 4 above). Indeed, if $\ddot{\vartheta}_{v i}, \dot{\vartheta}_{v i}$, and $\bar{z}_{i}-\vartheta_{v i} \rightarrow 0$, from the first equation in (44), it follows that also $e_{v i}$ converges to zero and this in turn implies that the second limit in (6) holds. To better see this, note that since

$$
e_{v i}=\sum_{j \in \mathcal{N}_{i}} a_{i j}\left[\vartheta_{v i}(t)-\vartheta_{v j}(t)+\int_{t-T_{j i}(t)}^{t} \dot{\vartheta}_{v j}(\sigma) d \sigma\right],
$$

defining $e_{v}:=\left[e_{v 1} \cdots e_{v N}\right]^{\top}$ and $\vartheta_{v}:=\left[\vartheta_{v 1} \cdots \vartheta_{v N}\right]$, respectively, we have

$$
e_{v}=\left(L \otimes I_{2}\right) \vartheta_{v}+\operatorname{col}\left[\sum_{j \in \mathcal{N}_{i}} a_{i j} \int_{t-T_{j i}(t)}^{t} \dot{\vartheta}_{v j}(\sigma) d \sigma\right] .
$$

Therefore, $e_{v i}=0$ and $\dot{\vartheta}_{v i}=0$ imply that $\left(L \otimes I_{2}\right) \vartheta_{v}=0$ which, in view of the properties of $L$, implies the existence of $\bar{z}_{c} \in \mathbb{R}^{2}$ such that $\vartheta_{v}=1_{N} \otimes \bar{z}_{c}$, or $\vartheta_{v i}=\bar{z}_{c}$ for all $i \leq N$. Hence, from the third equation in (44), we see that if $\ddot{\vartheta}_{v i}, \dot{\vartheta}_{v i}$, and $e_{v i} \rightarrow 0$, then

$$
\lim _{t \rightarrow \infty} \bar{z}_{i}(t)=\lim _{t \rightarrow \infty} \vartheta_{v i}(t) .
$$

and

$$
\lim _{t \rightarrow \infty} \bar{z}_{i}(t)=\bar{z}_{c} .
$$

The statement follows.

\section{SimULATION RESULTS}

In lack of an adequate set-up, we used the simulator Gazebo-ROS and the Robot Operating System (ROS) interface to evaluate the performance of our controller in a scenario that reproduces as closely as possible that of a laboratory experimental benchmark. Furthermore, for the sake of comparison, we also carried out numerical-integration simulations using Simulink of Matlab.

Gazebo-ROS is an efficient 3D dynamic simulator of robotic systems in indoor and outdoor environments. In contrast to pure numerical-integration based solvers of differential equations, Gazebo-ROS accurately emulates physical phenomena and dynamics otherwise neglected, such as friction, contact forces, actuator dynamics, slipping, etc. In addition, it offers high-fidelity robot and sensor simulations. 
For the test scenario we employed the model of a PIONEER 3-DX wheeled robot [64], available in Gazebo's library. It must be underlined that for this robot the center of mass is not located on the axis joining the two wheels' centers -cf. Fig. 1, as it is assumed at the basis of the developments in the previous sections. More precisely, in Equations (2) the functions $F_{v}$ and $F_{\omega}$ include Coriolis terms that are quadratic in the velocities, i.e., $\frac{r_{i}}{3} \omega_{i}^{2}$ on the left-hand side of Equation (2a) and $-\frac{r_{i} m_{i}}{3 I_{i}} \omega_{i} v_{i}$ on the left-hand side of Equation (2b). Akin to an actual experimentation set-up, these constitute dynamic effects not considered in the model for which the controller is validated analytically, nor in the simulations carried out with Simulink of Matlab.

Concretely, in the simulation scenario we consider six PIONEER 3D-X robots starting from initial postures as defined in the 2nd-4th columns of Table I, below.

TABLE I: Initial conditions

\begin{tabular}{cccccc}
\hline Index & $x_{i}[\mathbf{m}]$ & $y_{i}[\mathbf{m}]$ & $\theta_{i}[\mathbf{r a d}]$ & $\delta_{x i}[\mathbf{m}]$ & $\delta_{y i}[\mathbf{m}]$ \\
\hline 1 & 8 & 7 & 1.57 & 2 & 0 \\
\hline 2 & 2 & 13 & 0.0 & 1 & 2 \\
\hline 3 & 2 & 9 & -0.39 & -1 & 2 \\
\hline 4 & -2 & 6 & 0.39 & -2 & 0 \\
\hline 5 & 1 & 3 & -0.39 & -1 & -2 \\
\hline 6 & 4 & 4 & -0.39 & 1 & -2 \\
\hline
\end{tabular}

Also, an illustration of the robots in their initial postures is showed via a screenshot of the Gazebo-ROS simulator's user interface in Fig. 6, below.

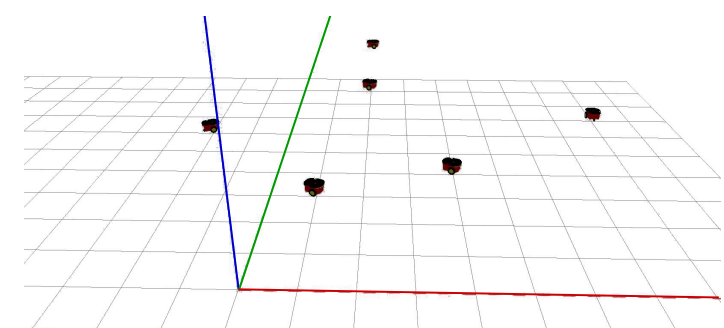

Fig. 6: Screenshot of the six PIONEER 3D-X robots' at their initial configuration, in the Gazebo-ROS simulator

It is assumed that the robots communicate over the undirected connected graph like the one illustrated in Fig. 7 and with piece-wise constant time-varying delays. For simplicity, all the time delays $T_{j i}(t)$ are taken equal; they are generated randomly following a normal distribution with mean $\mu=0.3$, variance $\sigma^{2}=0.0003$ and a sample time of $10 \mathrm{~ms}$ - see Fig. 8 . Such time delay (non-smooth but piece-wise continuous) does not satisfy Assumption 3 since its time-derivative is bounded only almost everywhere (that is, except at the points of discontinuity). However, it is considered in the simulations since it is closer to what is encountered in a real-world set-up [39]. Even though the technical Assumption 3 does not hold, full consensus is achieved (at least practically) in both the Matlab and the realistic Gazebo-ROS simulations. This hints at the fact that Assumption 3 might be relaxed in the analysis, albeit using a different controller —cf. [8].

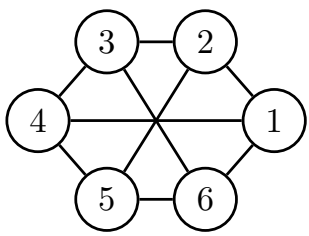

Fig. 7: Communication topology: undirected connected graph

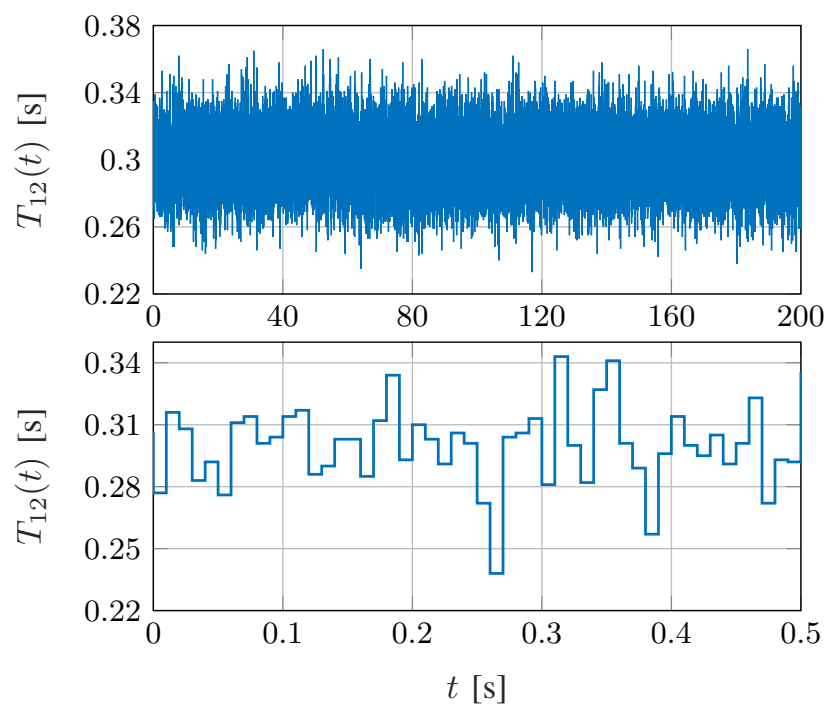

Fig. 8: Variable delay between the robot 1 and the received information from neighbor 2 .

The desired formation at rendezvous corresponds to a hexagon determined by desired offsets $\delta_{i}=\left(\delta_{x i}, \delta_{y i}\right)$ with respect the unknown center of the formation. These constants are presented in the last two columns of Table I — see Fig. 2 for an illustration of the target formation.

For a fair and meaningful comparison, the numerical simulations under Simulink of Matlab were performed using the information available on the PIONEER 3D-X robot, from the Gazebo-ROS simulator. For simplicity, it is assumed that all the robots have the same inertial and geometrical parameters given by $m=5.64 \mathrm{~kg}, I=3.115 \mathrm{~kg} \cdot \mathrm{m}^{2}, r=0.09 \mathrm{~m}$ and $R=0.157 \mathrm{~m}$

In both simulators, the control gains were set to $k_{v i}=1$, $k_{\omega i}=2, d_{v i}=3, p_{v i}=0.4, d_{\omega i}=2, p_{\omega i}=0.1$, for all $i \in[1,6]$. These values correspond to magnitudes compatible with the emulated physics of the PIONEER 3D-X robots in Gazebo-ROS and are chosen so that the poles of the 2nd-order system $\ddot{x}=-d_{(\cdot)} \dot{x}-p_{(\cdot)} x$ have negative real parts and the system have an over-damped step-response. The $\delta$-persistentlyexciting functions $\alpha_{i}$, for all $i \in[1,6]$, were taken as in (40) with $k_{\alpha i}=0.4$ and, for simplicity, (multi)periodic functions

$$
\begin{aligned}
\psi_{i}(t)= & 2.5+\sin (2 \pi t)+0.3 \cos (6 \pi t)-0.5 \sin (8 \pi t) \\
& -0.1 \cos (10 \pi t)+\sin (\pi t) \quad \forall i \leq 6 .
\end{aligned}
$$

Other parameters such as the sampling time, were taken equal.

As we mentioned above, however, certain physical phenomena as well as actuator and sensor dynamics, which are hard-coded in Gazebo-ROS, cannot be reproduced in Matlab. 
The consequence of this is clearly appreciated in the figures showed below. The results obtained with Gazebo-ROS are showed in Figs. 10, 12, 14, and 16. The results obtained using Simulink of Matlab are showed in Figs. 9, 12, 15, and 17.

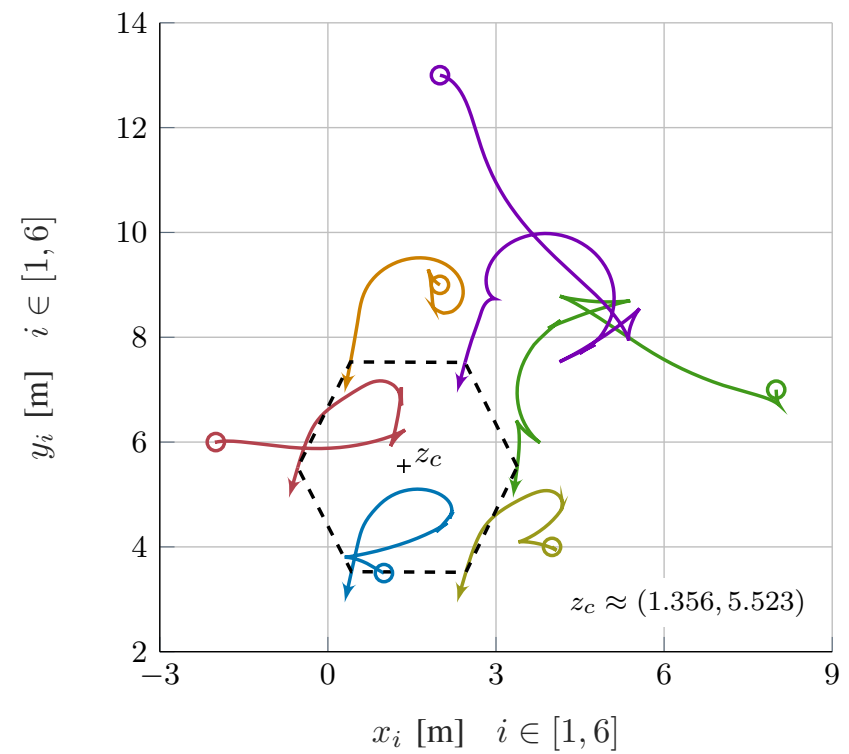

Fig. 9: Paths followed by the PIONEER 3D-X robots up to full formation consensus - Matlab simulation. A hexagonal formation is achieved with coinciding orientations (illustrated by arrows).

In both cases the robots appear to achieve consensus, i.e., to meet at a non-predefined rendezvous point in hexagonal formation and with common non-predefined orientation - see Figs. 9 and 10, as well as the screenshot of the final postures from Gazebo's graphical interface, Fig. 11. Under Matlab, the center of the formation is located at $(1.356,5.523)$, while under Gazebo-ROS it is at $(-3.242,-3.597)$. The consensual orientations are $\theta_{c} \approx-2.889 \mathrm{rad}$ under Gazebo-ROS and $\theta_{c} \approx-1.785 \mathrm{rad}$ under Matlab.

Both simulations illustrate that for networks of nonholonomic vehicles, the initial conditions do not determine the consensus point, as is the case of linear systems interconnected over static undirected connected graphs [1]. Indeed, the consensus point -in this case the center of the formation and the common orientation- does not correspond to the average of the initial conditions.

The consensus equilibrium heavily depends, as well, on the systems' nonlinear dynamics. This is clear both, in Fig. 9 which results of a Matlab simulation for a network of nonlinear systems modeled as in (1)-(2) with $F_{v}=F_{\omega}=0$, as well as in Fig. 10 which results from a more realistic simulation based on a model that emulates otherwise neglected Coriolis high-order terms, friction, sensor and actuator effects, etc. In addition, it appears fitting to recall that the controller, in both cases, is dynamic and time-varying.

Furthermore, it is clear from Figs. 9 and 10 that the results obtained with either simulator differ considerably in various manners. Obvious discrepancies lay in the position of the center of the consensus formation that is achieved, as well as in the paths followed by the robots.

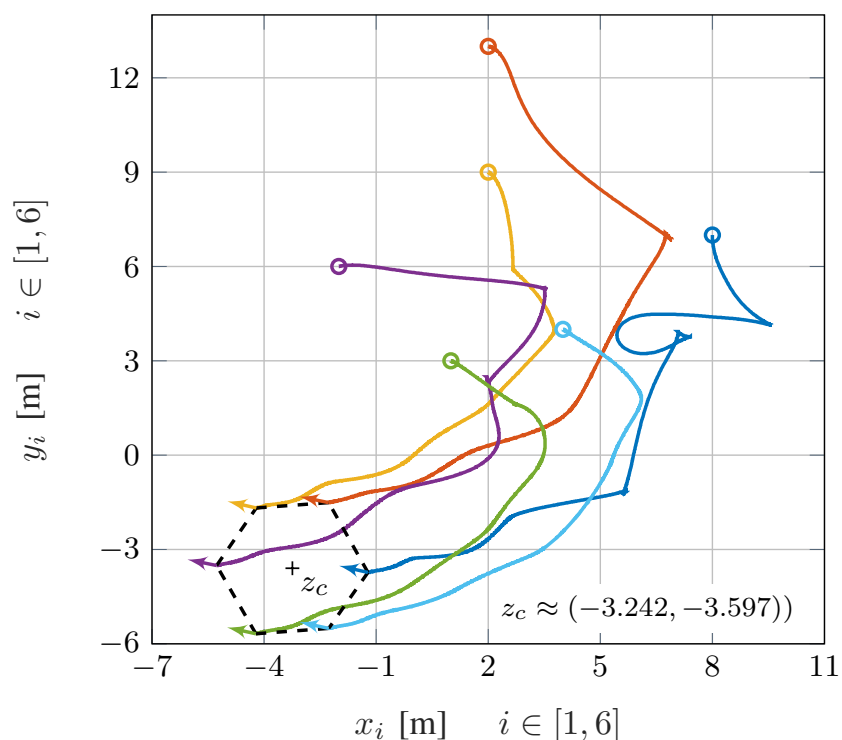

Fig. 10: Paths followed by the PIONEER 3D-X robots up to full formation consensus - Gazebo-ROS simulation. A hexagonal formation is achieved with coinciding orientations (illustrated by arrows).

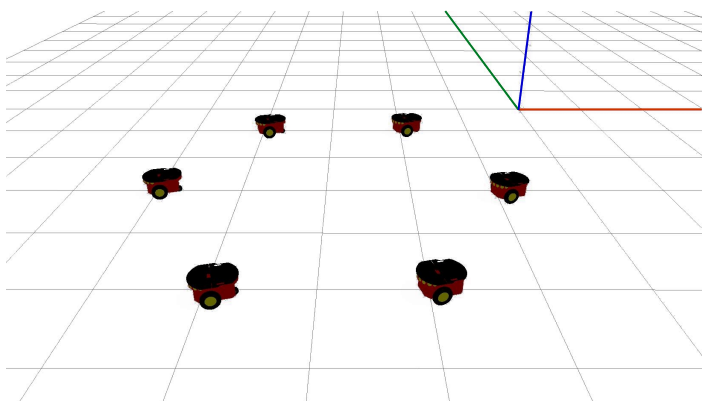

Fig. 11: Screenshot of the final configuration in the Gazebo-ROS simulator; the six robots achieving full consensus at the rendezvous point.

The differences in the transient behaviors for both simulations are even clearer in the plots of the consensus errors, which, for the purpose of graphic illustration, are defined as the difference between each robot's variables and the corresponding average:

$$
e_{z i}:=\bar{z}_{i}-\frac{1}{N} \sum_{j \in \mathcal{N}_{i}} \bar{z}_{j}, \quad e_{\theta i}:=\theta_{i}-\frac{1}{N} \sum_{j \in \mathcal{N}_{i}} \theta_{j}
$$

That is, the limits in (6) and (7) hold if the error trajectories $e_{z i}(t)$ and $e_{\theta i}(t)$ as defined above converge to zero, but the errors in (53) do not correspond to variables actually used by the controller nor measured for that matter.

In Fig. 12 one can appreciate that such errors do not actually tend to zero, but to a steady state-error - a keen observer will notice that the hexagon in Fig. 10 is actually not quite so. In contrast to this, in the simulation obtained using Matlab - see Fig. 13 - the errors converge to zero asymptotically, albeit slowly. The reason is that in the Gazebo-ROS simulation, after a transient, the amplitude of the input torques becomes considerably small in absolute value —see Fig. 16. 
The presence of a steady-state error and the persistency-ofexcitation effect in the controller maintain the input torques oscillating (periodically in this case due to the choice of $\psi_{i}(t)$ in (52)), but, physically, they result insufficient to overcome the robots' inertia and friction forces that oppose their forward and angular motions. In contrast to this, in Fig. 17 are showed the input torques obtained using Simulink of Matlab. A similar oscillating behavior is observed, but the torques vanish asymptotically - notice the order of magnitude in the plots on the right column in Fig. 17, in the range of milli-Nm - as the error-dependent persistency of excitation disappears.

It seems fitting to say at this point that the controller gains may be augmented, for instance, to increase the convergence speed, but such values may result incompatible with the robots' and actuators' physical limitations, so it is not done here to conserve a realistic setting.

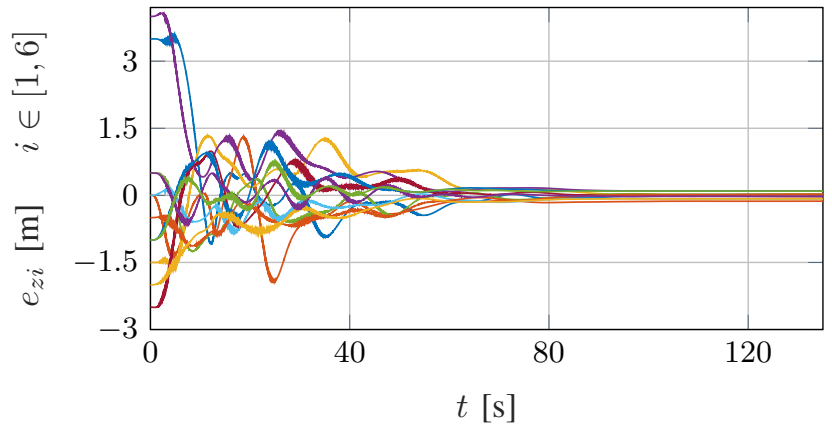

Fig. 12: Position consensus errors - Gazebo-ROS simulation.

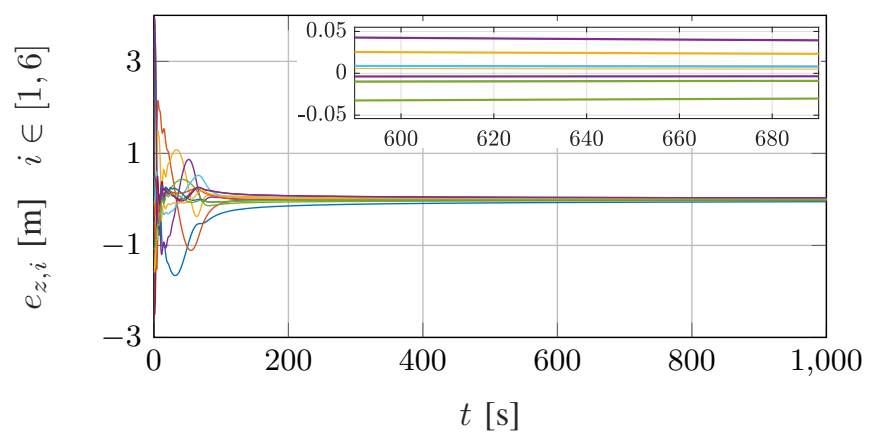

Fig. 13: Position consensus errors - Matlab simulation.

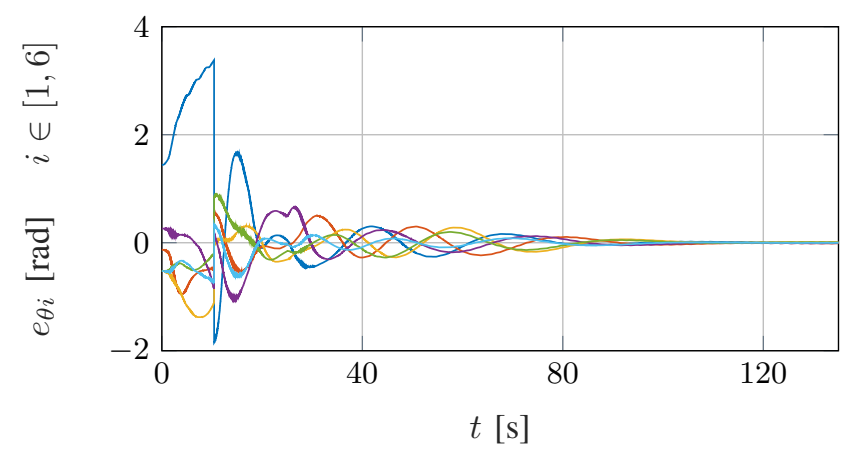

Fig. 14: Orientation consensus errors - Gazebo-ROS simulation. The consensus equilibrium $\theta_{c} \approx-2.889 \mathrm{rad}$

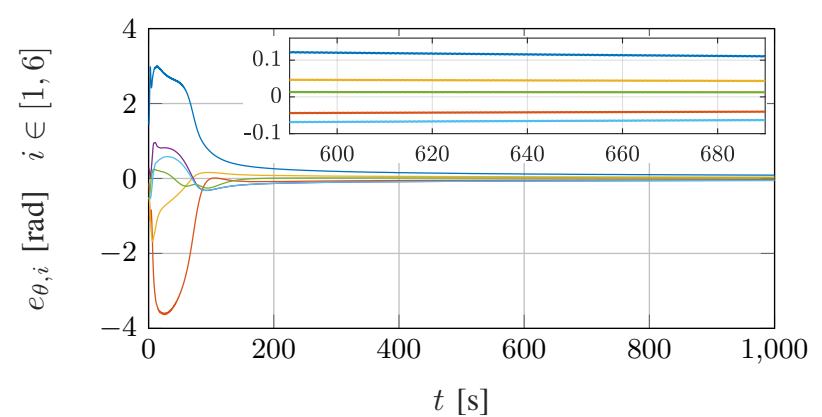

Fig. 15: Orientation consensus errors - Matlab simulation. The consensus equilibrium $\theta_{c} \approx-1.785 \mathrm{rad}$
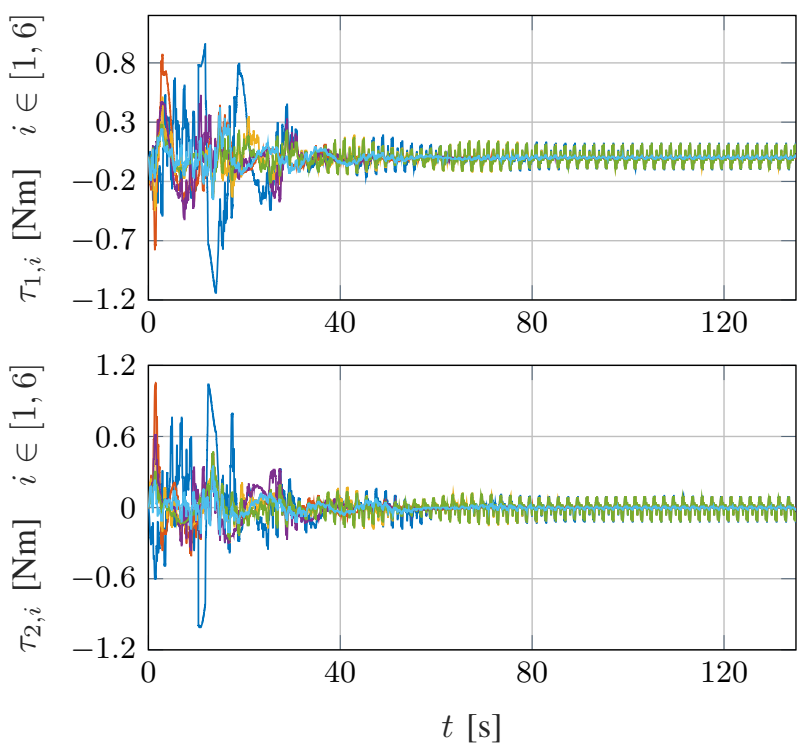

Fig. 16: Input torques - Gazebo-ROS simulation.
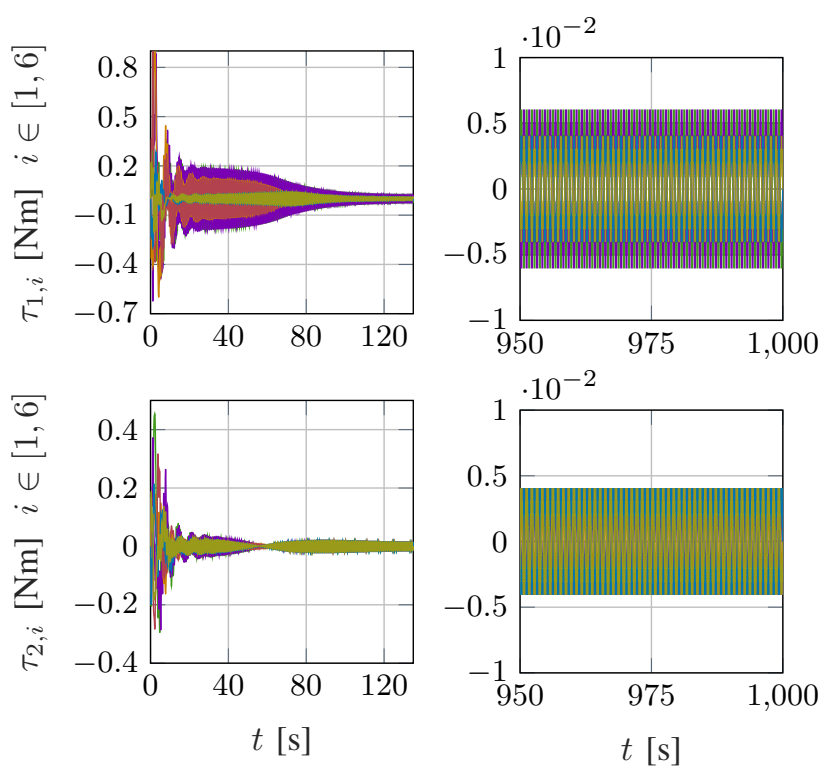

Fig. 17: Input torques - Matlab simulation. 


\section{CONCLUSIONS}

We have presented a simple dynamic output feedback controller for rendezvous of differential-drive robots. The novelty of our contributions lie in the lack of velocity measurements and the presence of time-varying delays. The controller that we propose has the neat physical interpretation of a second order mechanical system itself. In that regard, this technique may be a starting point for observerless control of multiagent systems under output feedback.

Even though the assumptions that our main results rely on are somewhat realistic, there are other hypotheses whose relaxing needs further study. Some pertain to the topology. In this work we assumed that the graph is undirected and static. The study of multiagent nonholonomic vehicles with less stringent hypotheses on the topology has been little addressed; for instance, with proximity constraints in [45] or under directed spanning-tree graph topologies in [44].

An intriguing aspect to investigate further is the influence of the nonlinear dynamics on consensus. Our theoretical results apply to the simplest of second-order nonholonomic systems, but other models in which the center of mass is not aligned with the axis joining the centers of the wheels should be investigated. The difficulty lies in the presence of highly nonlinear Lagrangian dynamics [33]-[36]. Yet, our numerical tests using the Gazebo-ROS simulator clearly show the effects of the nonlinearities in the consensus and the limitations of numerical algorithms bound to solving ordinary differential equations that describe over-simplified models, in which aspects such as unmodelled dynamics, friction and, actuator and sensor dynamics are neglected.

\section{REFERENCES}

[1] W. Ren and R. W. Beard, Distributed consensus in multivehicle cooperative control. Springer verlag, 2005.

[2] Z. Li, W. Ren, X. Liu, and M. Fu, "Consensus of multi-agent systems with general linear and lipschitz nonlinear dynamics using distributed adaptive protocols," IEEE Trans. on Automatic Control, vol. 58, no. 7, pp. 1786-1791, 2013.

[3] E. Panteley and A. Loría, "Synchronization and dynamic consensus of heterogeneous networked systems," IEEE Trans. on Automatic Control, vol. 62, no. 8, pp. 3758-3773, 2017.

[4] X. Zhao, X. Zheng, C. Ma, and R. Li, "Distributed consensus of multiple Euler-Lagrange systems networked by sampled-data information with transmission delays and data packet dropouts," IEEE Transactions on Automation Science and Engineering, vol. 14, no. 3, pp. 1440-1450, 2017.

[5] E. Nuño, "Consensus of Euler-Lagrange systems using only position measurements," IEEE Trans. on Control of Network Systems., vol. 5, no. 1, pp. 489-498, 2018.

[6] Z. Lin, B. Francis, and M. Maggiore, "Necessary and sufficient graphical conditions for formation control of unicycles," IEEE Trans. on Automatic Control, vol. 50, no. 1, pp. 121-127, 2005.

[7] E. Nuño, A. Loría, A. T. Hernández, M. Maghenem, and E. Panteley, "Distributed consensus-formation of force-controlled nonholonomic robots with time-varying delays," Automatica, no. 120, p. 109114, 2020.

[8] M. Maghenem, A. Loría, E. Nuño, and E. Panteley, "Distributed fullconsensus control of nonholonomic vehicles under non-differentiable measurement delays," IEEE Control Systems Letters, vol. 5, no. 1, pp. 97-102, 2021 .

[9] E. Montijano, J. Thunberg, X. Hu, and C. Sagüés, "Epipolar visual servoing for multirobot distributed consensus," IEEE Trans. on Robotics, vol. 29 , no. 5, pp. 1212-1225, 2013.

[10] S. Zhao, "Affine formation maneuver control of multiagent systems," IEEE Transactions on Automatic Control, vol. 63, no. 12, pp. 41404155, 2018 .
[11] A. Jadbabaie, J. Lin, and A. S. Morse, "Coordination of groups of mobile autonomous agents using nearest neighbor rules," IEEE Trans. on Automatic Control, vol. 48, no. 6, pp. 988-1001, 2003.

[12] D. Dimarogonas and K. Kyriakopoulos, "On the rendezvous problem for multiple nonholonomic agents," IEEE Transactions Automatic Control, vol. 52, pp. 916-922, May 2007.

[13] A. Roza, M. Maggiore, and L. Scardovi, "A Smooth Distributed Feedback for Global Rendezvous of Unicycles," IEEE Transactions Control Netw. Syst., vol. 5, pp. 640-652, Mar. 2018.

[14] L. Consolini, F. Morbidi, D. Prattichizzo, and M. Tosques, "On a class of hierarchical formations of unicycles and their internal dynamics," IEEE Trans. on Automatic Control, vol. 57, no. 4, pp. 845-859, 2012.

[15] J. A. Marshall, M. E. Broucke, and B. A. Francis, "Formations of vehicles in cyclic pursuit," IEEE Transactions on Automatic Control, vol. 49, no. 11, pp. 1963-1974, 2004.

[16] R. W. Brockett, "Asymptotic stability and feedback stabilization," Differential geometric control theory, vol. 27, no. 1, pp. 181-191, 1983.

[17] C. Yang, W. Xie, C. Lei, and B. Ma, "Smooth time-varying formation control of multiple nonholonomic agents," in Proceedings of the 2015 Chinese Intelligent Systems Conference, pp. 283-291, Springer, 2016.

[18] J. Jin and N. Gans, "Collision-free formation and heading consensus of nonholonomic robots as a pose regulation problem," Robotics and Autonomous Systems, vol. 95, no. 9, pp. 25-36, 2017.

[19] D. A. Lizárraga., "Obstructions to the existence of universal stabilizers for smooth control systems," Mathematics of Control, Signals and Systems, vol. 16, pp. 255-277, 2004.

[20] W. Dong, "Distributed observer-based cooperative control of multiple nonholonomic mobile agents," International Journal of Systems Science, vol. 43, no. 5, pp. 797-808, 2012.

[21] W. Mao, C. Wang, W. Chen, and X. Li, "Observer-based consensus design for multi-agent systems with unavailable velocities of leader and followers," in Proceedings of the 32nd Chinese Control Conference, pp. 7030-7033, 2013.

[22] X. Wang and Y. Hong, "Distributed observers for tracking a moving target by cooperative multiple agents with time delays," in 2009 ICCAS SICE, pp. 982-987, 2009.

[23] X. Liang, H. Wang, Y. Liu, W. Chen, and T. Liu, "Formation control of nonholonomic mobile robots without position and velocity measurements," IEEE Trans. on Robotics, vol. 34, no. 2, pp. 434-446, 2018.

[24] H. A. Poonawala, A. C. Satici, and M. W. Spong, "Leader-follower formation control of nonholonomic wheeled mobile robots using only position measurements," in 2013 9th Asian Control Conference (ASCC), pp. 1-6, June 2013.

[25] A. T. Hernández, A. Loría, E. Nuño, and E. Panteley, "Consensusformation control of nonholonomic robots without velocity measurements," in Proc. European Control Conference (ECC), (St. Petersburg, Russia), pp. 674-679, 2020.

[26] A. Loría, E. Panteley, and A. Teel, "A new persistency-of-excitation condition for UGAS of NLTV systems: Application to stabilization of nonholonomic systems," in Proc. 5th. European Contr. Conf., (Karlsrühe, Germany), pp. 1363-1368, 1999.

[27] N. Moshtagh and A. Jadbabaie, "Distributed geodesic control laws for flocking of nonholonomic agents," IEEE Transactions on Automatic Control, vol. 52, no. 4, pp. 681-686, 2007.

[28] B. Ning and Q. Han, "Prescribed finite-time consensus tracking for multiagent systems with nonholonomic chained-form dynamics," IEEE Transactions on Automatic Control, vol. 64, no. 4, pp. 1686-1693, 2019.

[29] P. Wang and B. Ding, "Distributed RHC for tracking and formation of nonholonomic multi-vehicle systems," IEEE Transactions on Automatic Control, vol. 59, no. 6, pp. 1439-1453, 2014.

[30] J. I. Neimark and F. A. Fufaev, Dynamics of Nonholonomic Systems, vol. 33. Providence, RI: A.M.S. Translations of Mathematical Monographs, 1972.

[31] Y. Cheng, R. Jia, H. Du, G. Wen, and W. Zhu, "Robust finite-time consensus formation control for multiple nonholonomic wheeled mobile robots via output feedback," International Journal of Robust and Nonlinear Control, vol. 28, no. 6, pp. 2082-2096, 2018.

[32] M. I. El-Hawwary and M. Maggiore, "Distributed circular formation stabilization for dynamic unicycles," IEEE Trans. on Automatic Control, vol. 58, no. 1, pp. 149-162, 2013.

[33] D. Lee, "Passive decomposition and control of nonholonomic mechanical systems," IEEE Trans. on Robotics, vol. 26, no. 6, pp. 978-992, 2010.

[34] K. D. Do, Z.-P. Jiang, and J. Pan, “A global output-feedback controller for simultaneous tracking and stabilization of unicycle-type mobile robots," IEEE Trans. on Robotics and Automation, vol. 20, no. 3, pp. 589-594, 2004. 
[35] J. Huang, C. Wen, W. Wang, and Z.-P. Jiang, "Adaptive output feedback tracking control of a nonholonomic mobile robot," Automatica, vol. 50, pp. 821-831, 2014.

[36] S. G. Tzafestas, Introduction to mobile robot control. Elsevier Inc, First ed., 2013.

[37] A. Loría, "From feedback to cascade-interconnected systems: Breaking the loop," in Proc. 47th. IEEE Conf. Decision Contr., (Cancun, Mex.), pp. 4109-4114, 2008

[38] M. Mesbahi and M. Egerstedt, Graph theoretic methods in multiagent networks. Princeton series in applied mathematics, Princeton: Princeton University Press, 2010.

[39] A. Abdessameud, I. Polushin, and A. Tayebi, "Synchronization of Lagrangian systems with irregular communication delays," IEEE Trans. on Automatic Control, vol. 59, no. 1, pp. 187-193, 2014.

[40] A. Abdessameud, A. Tayebi, and I. G. Polushin, "Leader-follower synchronization of euler-lagrange systems with time-varying leader trajectory and constrained discrete-time communication," IEEE Transactions on Automatic Control, vol. 62, pp. 2539-2545, May 2017.

[41] M. Maghenem, Stability and stabilization of networked time-varying systems. PhD thesis, Univ Paris Saclay, Gif sur Yvette, 2017. https://tel.archives-ouvertes.fr/tel-01596158.

[42] A. Abdessameud and A. Tayebi, "On consensus algorithms for doubleintegrator dynamics without velocity measurements and with input constraints," Systems \& Control Letters, vol. 59, no. 12, pp. 812-821, 2010.

[43] A. González, R. Aragüés, G. López-Nicolás, and C. Sagüés, "Stability analysis of nonholonomic multiagent coordinate-free formation control subject to communication delays," International Journal of Robust and Nonlinear Control, vol. 28, no. 14, pp. 4121-4138, 2018.

[44] H. A. Poonawala and M. W. Spong, "Preserving strong connectivity in directed proximity graphs," IEEE Trans. Automatic Control, vol. 62, no. 9, pp. 4392-4404, 2017.

[45] E. Restrepo, A. Loría, I. Sarras, and J. Marzat, "Stability and robustness of edge-agreement-based consensus protocols for undirected proximity graphs," International J. of Control, 2020.

[46] E. A. Barbashin and N. N. Krasovski1, “ Об устойчивости движения в целом," Dokl. Akad. Nauk. USSR, vol. 86, no. 3, pp. 453 456, 1952. Commonly (and wrongly) cited in English under: "On the stability of motion in the large"; Correct translation: "On the stability of motion in the whole".

[47] D. Hill and P. Moylan, "The stability of nonlinear dissipative systems," IEEE Trans. on Automatic Control, pp. 708-711, 1976.

[48] A. Bautista-Castillo, C. Lopez-Franco, and E. Nuño, "Consensus-based formation control for multiple nonholonomic robots," in 2016 IEEE International Autumn Meeting on Power, Electronics and Computing (ROPEC), IEEE, 2016.

[49] E. Nuño, I. Sarras, A. Loría, M. Maghenem, E. Cruz-Zavala, and E. Panteley, "Strict Lyapunov-Krasovskii functionals for undirected networks of Euler-Lagrange systems with time-varying delays," Syst. \& Contr. Letters, vol. 135, p. 104579, Jan 2020

[50] Y. Cao and W. Ren, Distributed Coordination of Multi-agent Networks: Emergent Problems, Models, and Issues. Springer-Verlag, 2011.

[51] Q. Hui, "Finite-time rendezvous algorithms for mobile autonomous agents," IEEE Trans. on Automatic Control, vol. 56, no. 1, pp. $207-$ 211, 2011.

[52] K. S. Narendra and A. M. Annaswamy, Stable adaptive systems. New Jersey: Prentice-Hall, Inc., 1989.

[53] E. Panteley, E. Lefeber, A. Loría, and H. Nijmeijer, "Exponential tracking of a mobile car using a cascaded approach," in IFAC Workshop on Motion Control, (Grenoble, France), pp. 221-226, 1998.

[54] C. Samson, "Time-varying stabilization of a car-like mobile robot," tech. rep., INRIA Sophia-Antipolis, 1990. In Proc. in advanced robot control 162 (Springer, Berlin, 1991).

[55] C. Samson, "Control of chained system: Application to path following and time-varying point stabilization of mobile robots," IEEE Trans. on Automatic Control, vol. 40, no. 1, pp. 64-77, 1995.

[56] W. E. Dixon, D. M. Dawson, F. Zhang, and E. Zergeroglu, "Global exponential tracking control of a mobile robot system via a PE condition," IEEE Trans. Systems, Man, and Cybernetics B, vol. 30, no. 1, pp. 129-142, 2000

[57] T. C. Lee, K. T. Song, C. H. Lee, and C. C. Teng, "Tracking control of unicycle-modeled mobile robots using a saturation feedback controller," IEEE Trans. on Control Systems Technology, vol. 9, no. 2, pp. 305-318, 2001.

[58] I. V. Burkov and A. T. Zaremba, "Dynamics of elastic manipulators with electric drives," Izv. Akad. Nauk SSSR Mekh. Tverd. Tela, vol. 22, no. 1, pp. 57-64, 1987. Engl. transl. in Mechanics of Solids, Allerton Press.
[59] M. Spong, "Modeling and control of elastic joint robots," ASME J. Dyn. Syst. Meas. Contr., vol. 109, pp. 310-319, 1987.

[60] E. Nuño and R. Ortega, "Achieving consensus of Euler-Lagrange agents with interconnecting delays and without velocity measurements via passivity-based control," IEEE Trans. on Control Systems Technology, vol. 26, no. 1, pp. 222-232, 2018.

[61] P. Ioannou and J. Sun, Robust adaptive control. New Jersey, USA: Prentice Hall, 1996.

[62] E. Fridman, "Tutorial on Lyapunov-based methods for time-delay systems," European Journal of Control, vol. 20, no. 6, pp. 271-283, 2014

[63] H. Wang, "Differential-cascade framework for consensus of networked lagrangian systems," Automatica, vol. 112, p. 108620, 2020.

[64] Génération Robots, "Pioneer P3-DX mobile robot." Accessed May 06, 2021. [Online]. https://www.generationrobots.com/en/402395-robotmobile-pioneer-3-dx.html.

[65] Barbălat, "Systèmes d'Équations Différentielles d'Oscillations Non Linéaires," Revue de Mathématiques Pures et Appliquées, vol. Vol. 4, no. 2, pp. 267-270, 1959. Académie de la République Populaire Roumaine (in French).

[66] B. Farkas and S.-A. Wegner, "Variations on Barbălat's lemma," The American Mathematical Monthly, vol. 123, no. 8, pp. 825-830, 2016.

[67] E. Nuño, I. Sarras, and L. Basañez, "Consensus in networks of nonidentical Euler-Lagrange systems using $\mathrm{P}+\mathrm{d}$ controllers," IEEE Trans. on Robotics, vol. 26, no. 6, pp. 1503-1508, 2013.

\section{APPENDIX}

A continuous function $t \mapsto \phi(t)$ is said to be in $\mathcal{L}_{\infty}$ (or to be bounded) if $\sup _{t>0}|\phi(t)|<\infty$. It is said to belong to $\mathcal{L}_{p}$ if $\left|\int_{0}^{t} \phi(s) d s\right|^{p}<\infty$.

Lemma A.1 (Barbălat [65]): If the function $t \mapsto f(t)$ is uniformly continuous and $\lim _{t \rightarrow \infty} \int_{0}^{t} f(s) d s$ exists and is finite, then $f(t) \rightarrow 0$ as $t \rightarrow \infty$.

Remark 8: The function $t \mapsto f(t)$ is uniformly continuous if $\dot{f} \in \mathcal{L}_{\infty}$, but the latter is not necessary.

Lemma A.2 (Lemma 3.2.5 in [61]): If for a function $t \mapsto$ $f(t), f$ and $\dot{f} \in \mathcal{L}_{\infty}$ and $f \in \mathcal{L}_{p}$ for some $p \in[1, \infty)$, then $f(t) \rightarrow 0$ as $t \rightarrow \infty$.

Lemma A.3 (Theorem 5 in [66]): Let $p \in[1, \infty)$ and $q \in$ $(1, \infty]$. Every function $t \mapsto f(t)$ such that $f \in \mathcal{L}_{p}$ and $\dot{f} \in \mathcal{L}_{q}$ tends to zero at infinity.

Proof of Claim 1: Consider the energy-like function defined in (45b). Its total derivative evaluated along the trajectories of (44) yields

$$
\begin{aligned}
\dot{\mathcal{E}}_{i}= & -d_{v i}\left|\dot{\vartheta}_{v i}\right|^{2}-p_{v i} \dot{\vartheta}_{v i}^{\top} e_{v i} \\
= & -d_{v i}\left|\dot{\vartheta}_{v i}\right|^{2}-p_{v i} \sum_{j \in \mathcal{N}_{i}} a_{i j} \dot{\vartheta}_{v i}^{\top}\left(\vartheta_{v i}-\vartheta_{v j}\right) \\
& -p_{v i} \sum_{j \in \mathcal{N}_{i}} a_{i j} \dot{\vartheta}_{v i}^{\top} \int_{t-T_{j i}(t)}^{t} \dot{\vartheta}_{v j}(\eta) d \eta,
\end{aligned}
$$

where, to obtain the second equation, we employed (36) and the fact that

$$
\vartheta_{v j}-\vartheta_{v j}\left(t-T_{j i}(t)\right)=\int_{t-T_{j i}(t)}^{t} \dot{\vartheta}_{v j}(\eta) d \eta
$$

Consider, next, the Lyapunov-Krasovsküi functional (45a). Its total derivative, under Assumption 2 and invoking [50, Lemma 6.1], yields

$\dot{\mathcal{V}}=-\sum_{i \leq N}\left[\frac{1}{p_{v i}} \dot{\mathcal{E}}_{i}+\sum_{j \in \mathcal{N}_{i}} a_{i j} \dot{\vartheta}_{v i}^{\top}\left[\vartheta_{v i}-\vartheta_{v j}\right]\right.$ 


$$
\left.-\frac{1}{2 \beta_{i}} \sum_{j \in \mathcal{N}_{i}} a_{i j}\left[\bar{T}_{j i}^{2}\left|\dot{\vartheta}_{v j}\right|^{2}-\bar{T}_{j i} \int_{t-\bar{T}_{j i}}^{t}\left|\dot{\vartheta}_{v j}\right|^{2}(\eta) d \eta\right]\right] .
$$

Hence, $\dot{\mathcal{V}}$ satisfies

$$
\begin{aligned}
\dot{\mathcal{V}}=- & \sum_{i \leq N}\left[\frac{d_{v i}}{p_{v i}}\left|\dot{\vartheta}_{v i}\right|^{2}+\sum_{j \in \mathcal{N}_{i}} a_{i j} \dot{\vartheta}_{v i}^{\top} \int_{t-T_{j i}(t)}^{t} \dot{\vartheta}_{v j}(\eta) d \eta\right. \\
& \left.-\frac{1}{2 \beta_{i}} \sum_{j \in \mathcal{N}_{i}} a_{i j}\left[\bar{T}_{j i}^{2}\left|\dot{\vartheta}_{v j}\right|^{2}-\bar{T}_{j i} \int_{t-\bar{T}_{j i}}^{t}\left|\dot{\vartheta}_{v j}\right|^{2}(\eta) d \eta\right]\right] .
\end{aligned}
$$

Applying Young's and Cauchy-Schwarz' inequalities on the second term of the right-hand side of $\dot{\mathcal{V}}_{i}$, we get

$$
\begin{aligned}
-\dot{\vartheta}_{v i}^{\top} \int_{t-T_{j i}(t)}^{t} \dot{\vartheta}_{v j}(\eta) d \eta & \leq \frac{\beta_{i}}{2}\left|\dot{\vartheta}_{v i}\right|^{2}+\frac{1}{2 \beta_{i}}\left|\int_{t-T_{j i}(t)}^{t} \dot{\vartheta}_{v j}(\eta) d \eta\right|^{2} \\
& \leq \frac{\beta_{i}}{2}\left|\dot{\vartheta}_{v i}\right|^{2}+\frac{\bar{T}_{j i}}{2 \beta_{i}} \int_{t-\bar{T}_{j i}}^{t}\left|\dot{\vartheta}_{v j}(\eta)\right|^{2} d \eta
\end{aligned}
$$

for any $\beta_{i}>0$. This yields

$$
\dot{\mathcal{V}} \leq-\sum_{i \leq N}\left[\left[\frac{d_{v i}}{p_{v i}}-\frac{\beta_{i}}{2} \ell_{i i}\right]\left|\dot{\vartheta}_{v i}\right|^{2}-\sum_{j \in \mathcal{N}_{i}} a_{i j} \frac{\bar{T}_{j i}^{2}}{2 \beta_{i}}\left|\dot{\vartheta}_{v j}\right|^{2}\right],
$$

where $\ell_{i i}$ is the $i$ th element in the diagonal of the Laplacian matrix - see (11). Then, proceeding as in [67] and defining $\sigma\left(\left|\dot{\vartheta}_{v i}\right|^{2}\right):=\left[\left|\dot{\vartheta}_{v 1}\right|^{2} \cdots\left|\dot{\vartheta}_{v N}\right|^{2}\right]^{\top}$ and

$A=\left[\begin{array}{cccc}\frac{d_{v 1}}{p_{v 1}}-\frac{\beta_{1}}{2} \ell_{11} & -\frac{\bar{T}_{21}^{2}}{2 \beta_{1}} a_{12} & \ldots & -\frac{\bar{T}_{N 1}^{2}}{2 \beta_{1}} a_{1 N} \\ -\frac{T_{12}}{2 \beta_{2}} a_{21} & \frac{d_{v 2}}{p_{v 2}}-\frac{\beta_{2}}{2} \ell_{22} & \ldots & -\frac{T_{N 2}^{2}}{2 \beta_{2}} a_{2 N} \\ \vdots & \vdots & \ddots & \vdots \\ -\frac{\bar{T}_{1 N}^{2}}{2 \beta_{N}} a_{N 1} & -\frac{\bar{T}_{2 N}^{2}}{2 \beta_{N}} a_{N 2} & \ldots & \frac{d_{v N}}{p_{v N}}-\frac{\beta_{N}}{2} \ell_{N N}\end{array}\right]$,

we obtain $\dot{\mathcal{V}} \leq-1_{N}^{\top} A \sigma\left(\left|\dot{\vartheta}_{v i}\right|^{2}\right)$ or, equivalently, Inequality (46). Thus, after (41), it follows that there exists $\lambda_{v i}>0$ such that

$$
\dot{\mathcal{V}} \leq-\sum_{i \leq N} \lambda_{v i}\left|\dot{\vartheta}_{v i}\right|^{2} \leq 0
$$

Integrating on both sides of the second inequality latter along the trajectories and observing that $\mathcal{V}$ is positive definite and radially unbounded with respect to $v_{i}, \dot{\vartheta}_{v i}, \vartheta_{v i}-\bar{z}_{i}$, and $\vartheta_{v i}-\vartheta_{v j}$, it follows that all corresponding trajectories are bounded. Using this and integrating on both sides of $\dot{\mathcal{V}} \leq$ $-\sum_{i \leq N} \lambda_{v i}\left|\dot{\vartheta}_{v i}\right|^{2}$ we obtain that $\dot{\vartheta}_{v i} \in \mathcal{L}_{2}$. This establishes the first part of the claim.

Now, from the above it follows that $e_{v i} \in \mathcal{L}_{\infty}$ and $\ddot{\vartheta}_{v i} \in$ $\mathcal{L}_{\infty}$ too and, after Barbălat's Lemma, that $\dot{\vartheta}_{v i} \rightarrow 0$. To see this more clearly, note that

$$
\begin{aligned}
\left|e_{v i}\right|^{2}= & \left|\sum_{j \in \mathcal{N}_{i}} a_{i j}\left[\vartheta_{v i}-\vartheta_{v j}+\int_{t-T_{j i}(t)}^{t} \dot{\vartheta}_{v j}(\sigma) d \sigma\right]\right|^{2} \\
\leq & \ell_{i i} \sum_{j \in \mathcal{N}_{i}} a_{i j}\left|\vartheta_{v i}-\vartheta_{v j}\right|^{2} \\
& +\ell_{i i} \sum_{j \in \mathcal{N}_{i}} a_{i j}\left|\int_{t-T_{j i}(t)}^{t} \dot{\vartheta}_{v j}(\sigma) d \sigma\right|^{2}
\end{aligned}
$$

$$
\begin{aligned}
\leq & \ell_{i i} \sum_{j \in \mathcal{N}_{i}} a_{i j}\left|\vartheta_{v i}-\vartheta_{v j}\right|^{2} \\
& +\ell_{i i} \sum_{j \in \mathcal{N}_{i}} a_{i j} \bar{T}_{j i} \int_{0}^{t}\left|\dot{\vartheta}_{v j}(\sigma)\right|^{2} d \sigma
\end{aligned}
$$

and all the terms on the right hand side of the last inequality are bounded. That $\dot{\vartheta}_{v i} \rightarrow 0$ follows invoking Lemma 2 above, which is a well-known consequence of Barbălat's Lemma.

Proof of Claim 2: Akin to the function $\mathcal{E}_{i}$ for the system $\Sigma_{v i}$, consider the energy-type function $\mathcal{H}_{i}$ defined in (47b), which is positive definite and radially unbounded in $\dot{\vartheta}_{\omega i}, \omega_{i}$, and $\vartheta_{\omega i}-\theta_{i}$. Its total time-derivative along the trajectories of (43) —with $\alpha_{i} \equiv 0$ - yields

$$
\begin{aligned}
\dot{\mathcal{H}}_{i}= & -d_{\omega i}\left|\dot{\vartheta}_{\omega i}\right|^{2}-p_{\omega i} \sum_{j \in \mathcal{N}_{i}} a_{i j} \dot{\vartheta}_{\omega i}^{\top}\left(\vartheta_{\omega i}-\vartheta_{\omega j}\right) \\
& -p_{\omega i} \sum_{j \in \mathcal{N}_{i}} a_{i j} \dot{\vartheta}_{\omega i}^{\top} \int_{t-T_{j i}(t)}^{t} \dot{\vartheta}_{\omega j}(\eta) d \eta .
\end{aligned}
$$

Then, proceeding as in the proof of Claim 1 we compute the total derivative of the Lyapunov-Krasovskì functional $\mathcal{W}$ defined in (47a) to obtain (47b). Under condition (42) there exists $\lambda_{\omega i}>0$ such that

$$
\dot{\mathcal{W}} \leq-\sum_{i \leq N} \lambda_{\omega i} \dot{\vartheta}_{\omega i}^{2}
$$

The first part of the Claim follows integrating along trajectories as in the Proof of Claim 1. That $e_{\omega i} \in \mathcal{L}_{\infty}$ follows from the bound

$$
\begin{aligned}
\left|e_{\omega i}\right|^{2} \leq & \ell_{i i} \sum_{j \in \mathcal{N}_{i}} a_{i j}\left|\vartheta_{\omega i}-\vartheta_{\omega j}\right|^{2} \\
& +\ell_{i i} \sum_{j \in \mathcal{N}_{i}} a_{i j} \bar{T}_{j i} \int_{0}^{t}\left|\dot{\vartheta}_{\omega j}(\sigma)\right|^{2} d \sigma
\end{aligned}
$$

-cf. (54), and observing that all the terms on the right-hand side are bounded. In turn, from (43c), we conclude that $\ddot{\vartheta}_{\omega i} \in$ $\mathcal{L}_{\infty}$. Thus, from Lemma A.2 we have $\dot{\vartheta}_{\omega i} \rightarrow 0$.

Proof of Claim 3: First we show that $\lim _{t \rightarrow \infty} \ddot{\vartheta}_{\omega i}(t)=0$ by invoking Barbălat's Lemma. To that end, note that since $\dot{\vartheta}_{\omega i} \rightarrow$ 0 ,

$$
\lim _{t \rightarrow \infty} \int_{0}^{t} \ddot{\vartheta}_{\omega i}(\sigma) d \sigma=\lim _{t \rightarrow \infty} \dot{\vartheta}_{\omega i}(t)-\dot{\vartheta}_{\omega i}(0)=-\dot{\vartheta}_{\omega i}(0) .
$$

On the other hand, $\ddot{\vartheta}_{\omega i}$ is uniformly continuous since $\vartheta_{\omega i}^{(3)}$ is bounded. To see this, note that

$$
\vartheta_{\omega i}^{(3)}=-d_{\omega i} \ddot{\vartheta}_{\omega i}-k_{\omega i}\left(\dot{\vartheta}_{\omega i}-\omega_{i}\right)-p_{\omega i} \dot{e}_{\omega i}
$$

where $\ddot{\vartheta}_{\omega i}, \dot{\vartheta}_{\omega i}, \omega_{i}$ were showed to be bounded above and so is $\dot{e}_{\omega i}$, in view of Assumption 3. Furthermore, from the latter and $\dot{\vartheta}_{\omega i} \rightarrow 0$ it also follows that $\dot{e}_{\omega i} \rightarrow 0$. Similarly, Assumption 3 and $\vartheta_{\omega i}^{(3)}, \ddot{\vartheta}_{\omega i}, \dot{\omega}_{i} \in \mathcal{L}_{\infty}$ imply, in turn, that $\vartheta_{\omega i}^{(4)} \in \mathcal{L}_{\infty}$. This implies that $\vartheta_{\omega i}^{(3)} \rightarrow 0$ and, in view of (55), also $\omega_{i} \rightarrow 0$. Thus,

$$
\lim _{t \rightarrow \infty} \int_{0}^{t} \dot{\omega}_{i}(\sigma) d \sigma=\lim _{t \rightarrow \infty} \omega_{i}(t)-\omega_{i}(0)=-\omega_{i}(0) .
$$

In addition, $\dot{\omega}_{i}$ is uniformly continuous because

$$
\ddot{\omega}_{i}=-k_{\omega i}\left(\omega_{i}-\dot{\vartheta}_{\omega i}\right)
$$


is bounded so, after Barbălat's Lemma it follows that $\dot{\omega}_{i} \rightarrow 0$ which, in view of (43), implies that (50) holds.

Also, from the third equation in (43), restricted to $\alpha_{i} \equiv$ 0 , we see that $\dot{\vartheta}_{\omega i}, \ddot{\vartheta}_{\omega i}$ and $\theta_{i}-\vartheta_{\omega i} \rightarrow 0$ implies that $\lim _{t \rightarrow \infty} e_{\omega i}(t)=0$ or, equivalently, that

$$
\lim _{t \rightarrow \infty} \sum_{j \in \mathcal{N}_{i}} a_{i j}\left[\vartheta_{\omega i}(t)-\vartheta_{\omega j}(t)+\int_{t-T_{j i}(t)}^{t} \dot{\vartheta}_{\omega j}(\sigma) d \sigma\right]=0 .
$$

Then, since $\dot{\vartheta}_{\omega i} \rightarrow 0$ and $T_{j i}(t)$ is globally bounded (see Assumption 3), we have

$$
\lim _{t \rightarrow \infty} \sum_{j \in \mathcal{N}_{i}} a_{i j}\left[\vartheta_{\omega i}(t)-\vartheta_{\omega j}(t)\right]=0
$$

which implies that $\lim _{t \rightarrow \infty} L \vartheta_{\omega}(t)=0$, where $\vartheta_{\omega}:=\operatorname{col}\left(\vartheta_{\omega i}\right) \in$ $\mathbb{R}^{N}$. Equivalently, there exists a constant $\theta_{c} \in \mathbb{R}$ such that $\lim _{t \rightarrow \infty} \vartheta_{\omega i}(t)=\theta_{c}$. This and (50) imply (49).

Proof of Claim 4: Consider again the second equation in (44). A simple inspection of the latter and of its first derivative, show that, since $\dot{v}_{i}, \ddot{v}_{i}, \dot{v}_{v i}$, and $v_{i} \rightarrow 0$,

$$
\varphi\left(\theta_{i}\right)^{\top}\left(\bar{z}_{i}-\vartheta_{v i}\right) \rightarrow 0, \quad \omega_{i} \varphi\left(\theta_{i}\right)^{\perp \top}\left(\bar{z}_{i}-\vartheta_{v i}\right) \rightarrow 0 .
$$

On the other hand, the solutions of the equation

$$
\varphi\left(\theta_{i}\right)^{\top}\left(\bar{z}_{i}-\vartheta_{v i}\right)=0
$$

are of the form $\left(\bar{z}_{i}-\vartheta_{v i}\right)=c_{1} \varphi\left(\theta_{i}\right)^{\perp}$ with $c_{1} \in \mathbb{R}$ while the solutions of the equation

$$
\omega_{i} \varphi\left(\theta_{i}\right)^{\perp \top}\left(\bar{z}_{i}-\vartheta_{v i}\right)=0
$$

are of the form $\left(\bar{z}_{i}-\vartheta_{v i}\right)=c_{2} \omega_{i} \varphi\left(\theta_{i}\right)$ with $c_{2} \in \mathbb{R}$. Therefore, (57) and (58) hold together if and only if $c \omega_{i} \varphi\left(\theta_{i}\right)=\varphi\left(\theta_{i}\right)^{\perp}$ with $c:=c_{1} / c_{2}$. In turn, the latter may hold only if either $c=0$ or $\omega_{i}=0$. Now, if $c=0$ then $\left(\bar{z}_{i}-\vartheta_{v i}\right)=0$. Thus, (56) imply that either $\left(\bar{z}_{i}-\vartheta_{v i}\right) \rightarrow 0$, which is to be showed, or $\omega_{i} \rightarrow 0$. In the latter case

$$
\lim _{t \rightarrow \infty} \int_{0}^{t} \dot{\omega}(s) d s=-\omega_{i}(0)<\infty
$$

and since $\ddot{\omega}_{i} \in \mathcal{L}_{\infty}$ we obtain, from Barbălat's Lemma, that $\dot{\omega}_{i} \rightarrow 0$. From a similar argument we conclude that $\ddot{\omega}_{i} \rightarrow 0$. Next, we show that $\omega_{i}, \dot{\omega}_{i}, \ddot{\omega}_{i} \rightarrow 0$ and $\dot{\psi}_{i} \not \supset 0$ - see (40)— imply together that $\left(\bar{z}_{i}-\vartheta_{v i}\right) \rightarrow 0$, so the proof ends. To that aim, we recall that the total derivative of $\mathcal{W}$ in (47a) along the trajectories of $\Sigma_{\omega}$ satisfies

$$
\dot{\mathcal{W}} \leq-\sum_{i \leq N}\left[\lambda_{\omega i} \dot{\vartheta}_{\omega i}^{2}-\frac{1}{p_{\omega i}} \alpha_{i} \omega_{i}\right]=:-\Psi
$$

in which we have dropped the arguments to avoid a cumbersome notation. Now, integrating on both sides of the inequality $\Psi \leq-\dot{\mathcal{W}}$, along the system's trajectories, and using the boundedness of $\mathcal{W}$, we see that $\Psi \in \mathcal{L}_{1}$. Furthermore, all the terms on the right-hand side of

$$
\dot{\Psi}=\sum_{i \leq N}\left[2 \lambda_{\omega i} \dot{\vartheta}_{\omega i} \ddot{\vartheta}_{\omega i}-\frac{1}{p_{\omega i}}\left[\dot{\alpha}_{i} \omega_{i}+\alpha_{i} \dot{\omega}_{i}\right]\right]
$$

depend, also, on bounded functions of time, so $\dot{\Psi} \in \mathcal{L}_{\infty}$. It follows, after Lemma A.3, that $\Psi \rightarrow 0$. Since $\alpha_{i} \in \mathcal{L}_{\infty}$ and $\omega_{i} \rightarrow 0$ it follows, in turn, that $\dot{\vartheta}_{\omega i} \rightarrow 0$. Therefore, since $\omega_{i}$, $\dot{\omega}_{i}, \ddot{\omega}_{i}, \dot{\vartheta}_{\omega i}, \dot{\vartheta}_{v i} \rightarrow 0$, all the terms in

$$
\begin{aligned}
\ddot{\omega}_{i}= & -k_{\omega i}\left(\omega_{i}-\dot{\vartheta}_{\omega i}\right)+k_{\alpha i} \dot{\psi}_{i}(t) \varphi\left(\theta_{i}\right)^{\perp \top}\left(\vartheta_{v i}-\bar{z}_{i}\right) \\
& -k_{\alpha i} \omega_{i} \psi_{i}(t) \varphi\left(\theta_{i}\right)^{\top}\left(\vartheta_{v i}-\bar{z}_{i}\right)+k_{\alpha i} \psi_{i}(t) \varphi\left(\theta_{i}\right)^{\perp \top} \dot{\vartheta}_{v i}
\end{aligned}
$$

tend to zero but in view of (56) and $\dot{\psi}_{i} \not \supset 0$ we necessarily have $\left(\vartheta_{v i}-\bar{z}_{i}\right) \rightarrow 0$. 